\title{
A conservative nonlocal convection-diffusion model and asymptotically compatible finite difference discretization
}

\author{
Hao Tian ${ }^{\mathrm{a}}$, Lili Ju ${ }^{\mathrm{b}, *}$, Qiang $\mathrm{Du}^{\mathrm{c}}$ \\ ${ }^{a}$ School of Mathematical Sciences, Ocean University of China, Qingdao, Shandong 266100, China. \\ ${ }^{b}$ Department of Mathematics, University of South Carolina, Columbia, SC 29208, USA. \\ ${ }^{c}$ Department of Applied Physics and Applied Mathematics, Columbia University, NY 10027, USA.
}

\begin{abstract}
In this paper, we first propose a nonlocal convection-diffusion model, in which the convection term is constructed in a special upwind manner so that mass conservation and maximum principle are maintained in any space dimension. The well-posedness of the proposed nonlocal model and its convergence to the classical local convection-diffusion model are established. A quadrature-based finite difference discretization is then developed to numerically solve the nonlocal problem and it is shown to be consistent and unconditionally stable. We further demonstrate that the numerical scheme is asymptotically compatible, that is, the approximate solutions converge to the exact solution of the corresponding local problem when $\delta \rightarrow 0$ and $h \rightarrow 0$. Numerical experiments are also performed to complement the theoretical analysis.
\end{abstract}

Keywords: Nonlocal convection-diffusion, mass conservation, maximum principle, quadrature-based finite difference, asymptotically compatible

\section{Introduction}

There have been much recent interests in nonlocal models given in the form of partial integral equations. Existing studies range from applications in nonlocal mechanics $[2,23,26,34,38,37,39$, 46], diffusion [24, 30, 33, 40], image analysis [1, 20, 21], biology [4, 31] to mathematical analysis and computational simulations $[3,5,8,9,11,12,14,18,22,45,27,41,48]$. Our goal here is to propose a new conservative nonlocal convection-diffusion model and discuss its effective numerical solution. While past studies on models of nonlocal diffusion have largely focused on pure symmetric processes where nonlocal interactions have no orientation bias towards any particular direction, the number of studies on nonlocal convection-diffusion models is also growing. In [13] a nonlinear nonlocal convection model is presented as a one-dimensional nonlocal balance law. A modified version was given in [10] based on the idea of monotone schemes. Several studies of fractional convectiondiffusion models may also be viewed as works on nonlocal models, see [19, 28] and the references

\footnotetext{
${ }^{\star} \mathrm{H}$. Tian's work is partially supported by Chinese Fundamental Research Funds for the Central Universities 201562012. L. Ju's work is partially supported by US National Science Foundation grant DMS-1521965 and US Department of Energy grants DE-SC0008087-ER65393 and DE-SC0016540. Q. Du's work is partially supported by US National Science Foundation grant DMS-1558744, US AFOSR MURI Center for Material Failure Prediction Through Peridynamics, and US Army Research Office MURI grant W911NF-15-1-0562.

${ }^{*}$ Corresponding author.

Email addresses: haot@ouc.edu.cn (Hao Tian), ju@math.sc.edu (Lili Ju), qd2125@columbia.edu (Qiang Du)
} 
cited therein. Research on the connections between the fractional, nonlocal and equations have been carried out in [8] and [7,6]. The nonlocal convection-diffusion model developed in [7] is globally mass conservative, but unfortunately does not have the maximum principle. By employing an upwind formulation of the nonlocal convection term, [43] proposed a new nonlocal convectiondiffusion model that shares several nice features of the local convection-diffusion including the maximum principle, but it does not guarantee mass conservation.

The main contributions of this work include the introduction and study of a new conservative and maximum principle preserving formulation, in any space dimension, of the nonlocal convectiondiffusion model with a special upwind feature, and the development and analysis of an asymptotically compatible discretization numerical scheme for solving the model. The basic structure of the model is consistent to the state-based approach proposed in [35] for the peridynamic model of nonlocal mechanics. Indeed, the range of nonlocal interactions in our nonlocal models is characterized by the so-called horizon parameter and the relevant nonlocal interaction kernel. Different from the typical symmetric and translation invariant kernels used in pure diffusion models, kernels in the nonlocal convection-diffusion are not symmetric, hence allowing convective effect. The term "upwind" mimics the same notion used in the numerical discretization of convective PDEs, that is, the upwind scheme that utilizes special stencils with respect to the convective velocity. In the nonlocal convection model, the upwind feature is naturally encoded in the kernel that is biased towards the direction of propagation of information, thus preserving important physics at the continuum level in arbitrary space dimension. We present both rigorous mathematical theory and careful study on numerical discretization. For the former, we discuss the total mass conservation, maximum principle and the basic well-posedness of the model. We also examine the convergence to the local convection-diffusion model as the nonlocal horizon parameter approaches to zero. On the numerical discretization, we focus on developing asymptotically compatible schemes, a concept developed in $[41,42]$ for the discretization of nonlocal models. Such a property is important for validation and verification as it ensures that the discrete numerical solutions of the nonlocal model can give the correct limit as the grid resolution gets refined and the nonlocal horizon shrinks. We show that on the discrete level, the quadrature-based finite difference discretization scheme for solving the nonlocal problem leads to a linear system associated with an $M$-matrix, which provides unique solvability and discrete maximum principle. We examine the consistency error of the scheme and also establish the asymptotic compatibility, that is, the approximate solutions converge to the exact solution of the corresponding local problem when $\delta \rightarrow 0$ and $h \rightarrow 0$ simultaneously in arbitrary fashion. Various numerical experiments with either a fixed $\delta$ or a fixed $\delta / h$ ratio are presented to validate the theoretical results.

The rest of the paper is organized as follows: some notations are first introduced in 1.1, then the formulation of the new conservative nonlocal convection-diffusion model and its mathematical theory are presented in Section 2. Next we propose and analyze a quadrature-based finite difference discretization scheme for the nonlocal model in Section 3. Some numerical experiments are reported in Section 4. Finally, some conclusions are given in Section 5.

\subsection{Notations}

We use the following notations throughout the paper. Let $\Omega_{s}$ be an open, connected and bounded domain in $\mathbb{R}^{n}$. The horizon parameter, denoted by $\delta>0$, represents the maximum distance within which nonlocal interaction can happen. Let $\Omega_{c}$ be an interaction domain for the specification of nonlocal boundary conditions [8]. For simplicity, we only consider the case where $\Omega_{c}$ is defined 
by $\Omega_{c}=\left\{\mathbf{x} \in \mathbb{R}^{n}-\Omega_{s} \mid \operatorname{dist}\left(\mathbf{x}, \Omega_{s}\right)<\delta\right\}$, and $\Omega=\Omega_{s} \cup \Omega_{c}$. For more discussions on nonlocal boundary conditions, we refer to [8] for a general set up and [17] and references cited therein for additional studies on Neumann (traction) type boundary conditions. Let $\boldsymbol{\alpha}(\mathbf{x}, \mathbf{y}): \mathbb{R}^{n} \times \mathbb{R}^{n} \rightarrow \mathbb{R}^{n}$ be a two-point vector mapping that is anti-symmetric, i.e., $\boldsymbol{\alpha}(\mathbf{y}, \mathbf{x})+\boldsymbol{\alpha}(\mathbf{x}, \mathbf{y})=0$. In the theory of nonlocal vector calculus $[8,9]$, the action of the nonlocal divergence operator on a locally integrable two-point vector function $\boldsymbol{\nu}(\mathbf{x}, \mathbf{y}), \mathcal{D}(\boldsymbol{\nu}): \mathbb{R}^{n} \rightarrow \mathbb{R}$, is defined as

$$
\mathcal{D}(\boldsymbol{\nu})(\mathbf{x}):=\int_{\mathbb{R}^{n}}(\boldsymbol{\nu}(\mathbf{x}, \mathbf{y})+\boldsymbol{\nu}(\mathbf{y}, \mathbf{x}))^{T} \boldsymbol{\alpha}(\mathbf{x}, \mathbf{y}) d \mathbf{y}, \quad \text { for } \mathbf{x} \in \mathbb{R}^{n}
$$

For any scalar function $u: \mathbb{R}^{n} \rightarrow \mathbb{R}$, the adjoint operator of the nonlocal divergence operator $\mathcal{D}$, $\mathcal{D}^{*}(u): \mathbb{R}^{n} \times \mathbb{R}^{n} \rightarrow \mathbb{R}^{n}$, is defined as

$$
\mathcal{D}^{*}(u)(\mathbf{x}, \mathbf{y})=(u(\mathbf{x})-u(\mathbf{y})) \boldsymbol{\alpha}(\mathbf{x}, \mathbf{y}), \quad \text { for } \mathbf{x}, \mathbf{y} \in \mathbb{R}^{n} .
$$

Without loss of generality, we assume in this paper that

$$
\alpha(\mathbf{x}, \mathbf{y})=\frac{\mathbf{y}-\mathbf{x}}{\|\mathbf{y}-\mathbf{x}\|}
$$

with $\|\cdot\|$ denoting the Euclidean metric, which is commonly used in peridynamics and many nonlocal models $[8,9,13]$.

\section{A conservative nonlocal convection-diffusion model}

\subsection{Model formulation}

We first consider the nonlocal diffusion term. For any $\mathbf{x}, \mathbf{y} \in \mathbb{R}^{n}$, let us define a symmetric two-point indicator function as

$$
1_{d}(\mathbf{x}, \mathbf{y})= \begin{cases}1, & \text { if }\|\mathbf{y}-\mathbf{x}\|<\delta \\ 0, & \text { otherwise }\end{cases}
$$

and a nonlocal influence function $\gamma_{d}(\|\mathbf{z}\|) \geq 0$ that is integrable in $B(\mathbf{0}, \delta)$ and satisfies

$$
\int_{B(\mathbf{0}, \delta)} z_{i} z_{j} \gamma_{d}(\|\mathbf{z}\|) d \mathbf{z}=\delta_{i j}
$$

Note that the above integral is written in terms of the Cartesian coordinates and $\delta_{i j}$ here represents the Kronecker delta. An example of kernels satisfying (2.2) can be given by

$$
\gamma_{d}(\|\mathbf{z}\|)=\frac{n\left(n+2-s_{d}\right)}{\beta_{n} \delta^{n+2-s_{d}}} \frac{1}{\|\mathbf{z}\|^{s_{d}}}
$$

where $\beta_{n}=\frac{n \pi^{n / 2}}{\Gamma(n / 2+1)}$ is the surface area of the unit ball $B(\mathbf{0}, 1)$ in $\mathbb{R}^{n}$ and $0 \leq s_{d}<n$ is a constant parameter that determines the kernel singularity of the nonlocal diffusion process.

Let a diffusion weight function be defined by

$$
w_{d}(\mathbf{x}, \mathbf{y})=1_{d}(\mathbf{x}, \mathbf{y}) \gamma_{d}(\|\mathbf{y}-\mathbf{x}\|) .
$$


We remark that $w_{d}$ is used to measure the extent of influence of the nonlocal diffusion between $\mathbf{x}$ and $\mathbf{y}$. Given a scalar function $a \in L^{\infty}(\Omega)$ with

$$
0<a_{0} \leq a(\mathbf{x}) \leq a_{1}<\infty, \quad \forall \mathbf{x} \in \Omega
$$

for some positive constants $a_{0}$ and $a_{1}$, we define the corresponding two-point coefficient function $\Theta: \Omega \times \Omega \rightarrow \mathbb{R}$ for the nonlocal diffusion as

$$
\boldsymbol{\Theta}(\mathbf{x}, \mathbf{y})=a(\mathbf{x}) w_{d}(\mathbf{x}, \mathbf{y}) .
$$

Finally we construct the nonlocal (isotropic) diffusion operator, $\mathcal{L}^{\text {diff }}$, as

$$
\mathcal{L}^{\operatorname{diff}} u(\mathbf{x})=\mathcal{D}\left(\boldsymbol{\Theta} \mathcal{D}^{*} u\right)(\mathbf{x}), \quad \text { for } \mathbf{x} \in \Omega_{s} .
$$

Next we turn to the nonlocal convection term. Given a velocity function $\boldsymbol{b} \in\left(L^{\infty}(\Omega)\right)^{n}$ satisfying

$$
\|\boldsymbol{b}(\mathbf{x})\| \leq \hat{b}<\infty, \quad \forall \mathbf{x} \in \Omega
$$

for some positive constant $\hat{b}$, let us define the two-point indicator function $1_{c}(\mathbf{x}, \mathbf{y})$ as

$$
1_{c}(\mathbf{x}, \mathbf{y})= \begin{cases}1, & \text { if }\|\mathbf{y}-\mathbf{x}\|<\delta \text { and } \boldsymbol{b}(\mathbf{x})^{T}(\mathbf{y}-\mathbf{x})>0 \\ 0, & \text { otherwise }\end{cases}
$$

which is clearly velocity-dependent and usually non-symmetric. Let us define another influence function $\gamma_{c}(\|\mathbf{z}\|) \geq 0$ that is integrable in $B(\mathbf{0}, \delta)$ and satisfies

$$
\int_{B(\mathbf{0}, \delta)} \frac{z_{i} z_{j}}{2\|\mathbf{z}\|} \gamma_{c}(\|\mathbf{z}\|) d \mathbf{z}=\delta_{i j}
$$

Similar as before, an example can be given by

$$
\gamma_{c}(\|\mathbf{z}\|)=\frac{2 n\left(n+1-s_{c}\right)}{\beta_{n} \delta^{n+1-s_{c}}} \frac{1}{\|\mathbf{z}\|^{s_{c}}}
$$

where $0 \leq s_{c}<n$ is a constant parameter that determines the kernel singularity of the nonlocal convection process.

We then define a convection weight function as

$$
w_{c}(\mathbf{x}, \mathbf{y})=1_{c}(\mathbf{x}, \mathbf{y}) \gamma_{c}(\|\mathbf{y}-\mathbf{x}\|) \text {. }
$$

Finally a two-point vector function $\boldsymbol{\mu}: \Omega \times \Omega \rightarrow \mathbb{R}^{n}$ is defined as

$$
\boldsymbol{\mu}(\mathbf{x}, \mathbf{y})=\boldsymbol{b}(\mathbf{x}) w_{c}(\mathbf{x}, \mathbf{y})
$$

and consequently the nonlocal convection operator, $\mathcal{L}^{\text {conv }}$, is constructed as

$$
\mathcal{L}^{\text {conv }} u(\mathbf{x})=\mathcal{D}(\boldsymbol{\mu} u)(\mathbf{x}) .
$$

We note that the indicator function $1_{c}(\mathbf{x}, \mathbf{y})$ is defined in a special upwind sense, leading to bias in the nonlocal interaction with respect to the direction of propagation of information. Such 
an approach plays a crucial role in the proposed nonlocal convection-diffusion model in terms of guaranteeing the mass conservation and preserving the maximum principle.

We now summarize the new nonlocal convection-diffusion model as follows:

$$
\left\{\begin{aligned}
\mathcal{L} u & =f, & & \mathbf{x} \in \Omega_{s}, \\
u & =g, & & \mathbf{x} \in \Omega_{c},
\end{aligned}\right.
$$

where $\mathcal{L} u=\mathcal{L}^{\text {diff }} u+\mathcal{L}^{\text {conv }} u, f \in L^{2}\left(\Omega_{s}\right)$ and $g \in L^{2}\left(\Omega_{c}\right)$. The problem (2.13) is an integral equation in $\Omega_{s}$ along with a nonlocal volumetrically constrained Dirichlet boundary condition in the interaction domain $\Omega_{c}[8]$.

By substituting $\mathcal{L}$ in (2.13) with an operator that is a sum of both (2.6) and (2.12) and noticing that $\boldsymbol{\alpha}(\mathbf{x}, \mathbf{y})^{T} \boldsymbol{\alpha}(\mathbf{x}, \mathbf{y})=1$, we can get the formulation of the nonlocal convection-diffusion equation as follows: for any $\mathbf{x} \in \Omega_{s}$,

$$
\begin{aligned}
& \left.-\int_{\Omega}(a(\mathbf{x})+a(\mathbf{y}))(u(\mathbf{y})-u(\mathbf{x})) \omega_{d}(\mathbf{x}, \mathbf{y})\right) d \mathbf{y} \\
& \quad+\int_{\Omega}\left(\boldsymbol{b}(\mathbf{x}) u(\mathbf{x}) \omega_{c}(\mathbf{x}, \mathbf{y})+\boldsymbol{b}(\mathbf{y}) u(\mathbf{y}) \omega_{c}(\mathbf{y}, \mathbf{x})\right)^{T} \frac{\mathbf{y}-\mathbf{x}}{\|\mathbf{y}-\mathbf{x}\|} d \mathbf{y}=f(\mathbf{x}) .
\end{aligned}
$$

Clearly, with our choice of $\Omega_{c}=\left\{\mathbf{x} \in \mathbb{R}^{n}-\Omega_{s} \mid \operatorname{dist}\left(\mathbf{x}, \Omega_{s}\right)<\delta\right\}$, the integration domain $\Omega$ in the above equation (2.14) can be equivalently replaced by $B(\mathbf{x}, \delta)$. We also note that the corresponding local convection-diffusion model is given by

$$
\left\{\begin{aligned}
-\nabla \cdot(a \nabla u)+\nabla \cdot(\boldsymbol{b} u) & =f, & & \mathbf{x} \in \Omega, \\
u & =g, & & \mathbf{x} \in \partial \Omega .
\end{aligned}\right.
$$

Remark 1. Naturally we may use other types of two-point coefficient functions for the nonlocal kernel functions, for instance, $\mathbf{\Theta}(\mathbf{x}, \mathbf{y})=a\left(\frac{\mathbf{x}+\mathbf{y}}{2}\right) w_{d}(\mathbf{x}, \mathbf{y})$ for the diffusion, and for the convection $\boldsymbol{\mu}(\mathbf{x}, \mathbf{y})=\boldsymbol{b}\left(\frac{\mathbf{x}+\mathbf{y}}{2}\right) w_{c}(\mathbf{x}, \mathbf{y})$ with $\boldsymbol{b}\left(\frac{\mathbf{x}+\mathbf{y}}{2}\right)^{T}(\mathbf{y}-\mathbf{x})>0$ being used in the definition of $1_{c}(\mathbf{x}, \mathbf{y})$ in $(2.7)$. Such choices may be related to similar choices made in the finite difference or finite volume setting where one may either use the velocity at the cell-boundary or at the cell center.

Let us take a careful look at the influence regions of the two-point indicator functions $1_{d}(\mathbf{x}, \mathbf{y})$ and $1_{c}(\mathbf{x}, \mathbf{y})$ (see their definitions in (2.1) and (2.7)). For any given subdomain $U \in \Omega$, we denote its characteristic function as $1_{U}$ such that $1_{U}(\mathbf{x})=1$ if $\mathbf{x} \in U$ and $1_{U}(\mathbf{x})=0$ otherwise. For the nonlocal diffusion operator, the influence region of $1_{d}(\mathbf{x}, \mathbf{y})$ with a fixed point $\mathbf{x} \in \Omega_{s}$ is the ball $B(\mathbf{x}, \delta)$ (see the left panel of Fig 1$)$. We then have $1_{d}(\mathbf{x}, \mathbf{y})=1_{d}(\mathbf{y}, \mathbf{x})=1_{B(\mathbf{x}, \delta)}(\mathbf{y})=1_{B(\mathbf{y}, \delta)}(\mathbf{x})$ for any $\mathbf{x}, \mathbf{y} \in \Omega$. For the nonlocal convection operator, the influence region of $1_{c}(\mathbf{x}, \mathbf{y})$ with fixed $\mathbf{x}$ is the half-ball, $\widetilde{H B}(\mathbf{x}, \delta)$ (see the middle panel of Fig 1), i.e., $1_{c}(\mathbf{x}, \mathbf{y})=1_{\widetilde{H B}(\mathbf{x}, \delta)}(\mathbf{y})$ if $\|\boldsymbol{b}(\mathbf{x})\| \neq 0$. Note that the long axis of $\widetilde{H B}(\mathbf{x}, \delta)$ is perpendicular to $\boldsymbol{b}(\mathbf{x})$, the velocity vector at $\mathbf{x}$. However, $1_{c}(\mathbf{x}, \mathbf{y})$ is not symmetric with respect to $\mathbf{x}$ and $\mathbf{y}$ if $\mathbf{b}$ is not a constant velocity. According to (2.7), we have

$$
1_{c}(\mathbf{y}, \mathbf{x})= \begin{cases}1, & \text { if }\|\mathbf{y}-\mathbf{x}\|<\delta \text { and } \boldsymbol{b}(\mathbf{y})^{T}(\mathbf{x}-\mathbf{y})>0 \\ 0, & \text { otherwise. }\end{cases}
$$

Let $H B(\mathbf{x}, \delta)$ denote the influence region of $1_{c}(\mathbf{y}, \mathbf{x})$ for fixed $\mathbf{x}$, i.e., $1_{c}(\mathbf{y}, \mathbf{x})=1_{H B(\mathbf{x}, \delta)}(\mathbf{y})$. It is clear from (2.16) that $H B(\mathbf{x}, \delta)$ is determined by the velocity field inside $B(\mathbf{x}, \delta)$, see the right panel of Fig 1 for an illustration. Let us define $\widehat{H B}(\mathbf{x}, \delta)=B(\mathbf{x}, \delta)-\widetilde{H B}(\mathbf{x}, \delta)$, which gives the reflected half-ball of $\widetilde{H B}(\mathbf{x}, \delta)$. 

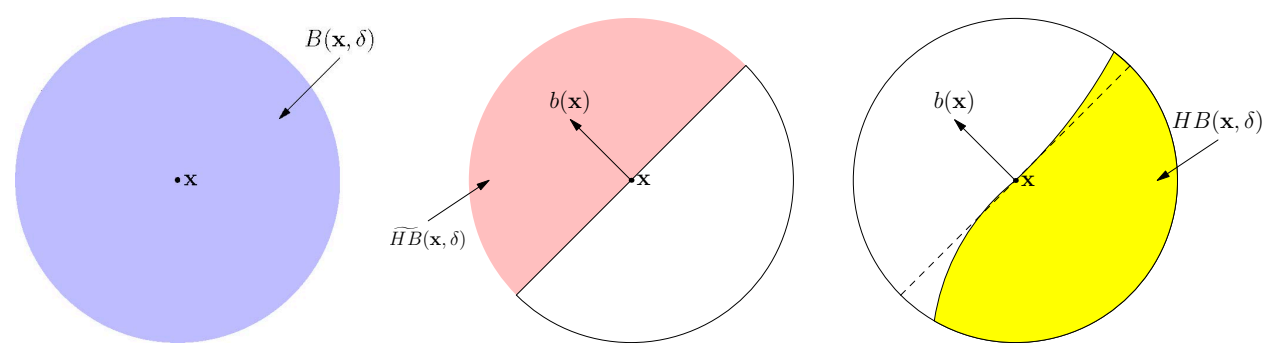

Figure 1: Illustration of the influence regions of the indicator functions for the nonlocal convectiondiffusion model (2.13). Left: $B(\mathbf{x}, \delta)$; middle: $\widetilde{H B}(\mathbf{x}, \delta)$; right: $H B(\mathbf{x}, \delta)$.

\subsection{Mass conservation}

Unlike the nonlocal convection-diffusion equation proposed in [43] that results in an upwind but non-conservative model, the new nonlocal convection-diffusion model (2.13) satisfies the global mass conservation as shown below. Let us set

$$
\gamma(\mathbf{x}, \mathbf{y})=(a(\mathbf{x})+a(\mathbf{y})) \omega_{d}(\mathbf{x}, \mathbf{y})+\boldsymbol{b}(\mathbf{x})^{T} \frac{\mathbf{y}-\mathbf{x}}{\|\mathbf{y}-\mathbf{x}\|} \omega_{c}(\mathbf{x}, \mathbf{y})
$$

which can be regarded as the kernel function (nonsymmetric) for the whole nonlocal convectiondiffusion operator. Since $(a(\mathbf{x})+a(\mathbf{y})) 1_{d}(\mathbf{x}, \mathbf{y}) \geq 0$ and $\boldsymbol{b}(\mathbf{x})^{T}(\mathbf{y}-\mathbf{x}) 1_{c}(\mathbf{x}, \mathbf{y}) \geq 0$, we have $\boldsymbol{\gamma}(\mathbf{x}, \mathbf{y}) \geq$ 0 for any $\mathbf{x}, \mathbf{y} \in \Omega$. It is easy to verify that the convection-diffusion nonlocal operator $\mathcal{L}$ can be rewritten as

$$
\mathcal{L} u(\mathbf{x})=\int_{\Omega} u(\mathbf{x}) \gamma(\mathbf{x}, \mathbf{y})-u(\mathbf{y}) \gamma(\mathbf{y}, \mathbf{x}) d \mathbf{y} .
$$

By integrating both sides of (2.18) on $\Omega_{s}$, we then have

$$
\begin{aligned}
\int_{\Omega_{s}} \mathcal{L} u(\mathbf{x}) d \mathbf{x}= & \int_{\Omega_{s}} \int_{\Omega_{c} \cup \Omega_{s}} u(\mathbf{x}) \gamma(\mathbf{x}, \mathbf{y})-u(\mathbf{y}) \gamma(\mathbf{y}, \mathbf{x}) d \mathbf{y} d \mathbf{x} \\
= & \int_{\Omega_{s}} \int_{\Omega_{c}} u(\mathbf{x}) \gamma(\mathbf{x}, \mathbf{y})-u(\mathbf{y}) \gamma(\mathbf{y}, \mathbf{x}) d \mathbf{y} d \mathbf{x} \\
& \quad+\int_{\Omega_{s}} \int_{\Omega_{s}} u(\mathbf{x}) \gamma(\mathbf{x}, \mathbf{y})-u(\mathbf{y}) \gamma(\mathbf{y}, \mathbf{x}) d \mathbf{y} d \mathbf{x} .
\end{aligned}
$$

By interchanging the variable $\mathbf{x}$ and $\mathbf{y}$, we have

$$
\begin{aligned}
& \int_{\Omega_{s}} \int_{\Omega_{s}} u(\mathbf{x}) \boldsymbol{\gamma}(\mathbf{x}, \mathbf{y})-u(\mathbf{y}) \boldsymbol{\gamma}(\mathbf{y}, \mathbf{x}) d \mathbf{y} d \mathbf{x} \\
& \quad=\frac{1}{2}\left(\int_{\Omega_{s}} \int_{\Omega_{s}}[u(\mathbf{x}) \boldsymbol{\gamma}(\mathbf{x}, \mathbf{y})-u(\mathbf{y}) \boldsymbol{\gamma}(\mathbf{y}, \mathbf{x})]+[u(\mathbf{y}) \boldsymbol{\gamma}(\mathbf{y}, \mathbf{x})-u(\mathbf{x}) \boldsymbol{\gamma}(\mathbf{x}, \mathbf{y})] d \mathbf{y} d \mathbf{x}\right) \\
& \quad=0 .
\end{aligned}
$$

This means that the flux from $\Omega_{s}$ into itself is zero, i.e., there is no net self-interaction [9]. Thus it holds that

$$
\int_{\Omega_{s}} \mathcal{L} u(\mathbf{x}) d \mathbf{x}=\int_{\Omega_{s}} \int_{\Omega_{c}} u(\mathbf{x}) \gamma(\mathbf{x}, \mathbf{y})-u(\mathbf{y}) \gamma(\mathbf{y}, \mathbf{x}) d \mathbf{y} d \mathbf{x}
$$


and we obtain the mass conservation of the proposed nonlocal model as

$$
\int_{\Omega_{s}} \int_{\Omega_{c}} u(\mathbf{x}) \boldsymbol{\gamma}(\mathbf{x}, \mathbf{y})-u(\mathbf{y}) \boldsymbol{\gamma}(\mathbf{y}, \mathbf{x}) d \mathbf{y} d \mathbf{x}=\int_{\Omega_{s}} f(\mathbf{x}) d \mathbf{x} .
$$

Here, the left hand side term is the nonlocal flux from the interior domain $\Omega_{s}$ to the interaction domain $\Omega_{c}$ while the right hand side term is the source from $\Omega_{s}$, and putting together on the same side of the equation, they cancel out each other.

\subsection{Maximum principle}

The nonlocal convection operator (2.12) can be further split into two parts as

$$
\begin{aligned}
\mathcal{L}^{\text {conv }} u(\mathbf{x}) & =\int_{\Omega}\left(\boldsymbol{b}(\mathbf{y}) u(\mathbf{y}) \omega_{c}(\mathbf{y}, \mathbf{x})+\boldsymbol{b}(\mathbf{x}) u(\mathbf{x}) \omega_{c}(\mathbf{x}, \mathbf{y})\right)^{T} \frac{\mathbf{y}-\mathbf{x}}{\|\mathbf{y}-\mathbf{x}\|} d \mathbf{y} \\
& =\int_{\Omega}(u(\mathbf{y})-u(\mathbf{x})) \omega_{c}(\mathbf{y}, \mathbf{x}) \boldsymbol{b}(\mathbf{y})^{T} \frac{\mathbf{y}-\mathbf{x}}{\|\mathbf{y}-\mathbf{x}\|} d \mathbf{y}+u(\mathbf{x}) \mathcal{D}(\boldsymbol{\mu})(\mathbf{x})
\end{aligned}
$$

with

$$
\mathcal{D}(\boldsymbol{\mu})(\mathbf{x})=\int_{\Omega}\left(\boldsymbol{b}(\mathbf{y}) \omega_{c}(\mathbf{y}, \mathbf{x})+\boldsymbol{b}(\mathbf{x}) \omega_{c}(\mathbf{x}, \mathbf{y})\right)^{T} \frac{\mathbf{y}-\mathbf{x}}{\|\mathbf{y}-\mathbf{x}\|} d \mathbf{y}
$$

which is analogous to its local counterpart

$$
\nabla \cdot(\boldsymbol{b} u)=\boldsymbol{b} \cdot \nabla u+u \nabla \cdot \boldsymbol{b} .
$$

It is known that the local convection-diffusion equation (2.15) satisfies the maximum principle if $\nabla \cdot \boldsymbol{b} \geq 0$, see [25, 29, 44, 47]. In comparison, the proposed nonlocal convection-diffusion operator satisfies a similar property stated below.

Theorem 1. (Maximum principle) Suppose that $\mathcal{D}(\boldsymbol{\mu}) \geq 0$ in $\Omega_{s}$ and $\mathcal{L} u$ is well-defined in $\Omega_{s}$. If $\mathcal{L} u<0$ in $\Omega_{s}$, then a nonnegative maximum of $u$ is only attained in the interaction domain $\Omega_{c}$.

Proof. We show that a nonnegative maximum can not be attained in $\Omega_{s}$ by contradiction. Assume that $u$ attains a nonnegative maximum at $\mathbf{x}_{0} \in \Omega_{s}$, i.e., $u\left(\mathbf{x}_{0}\right)=\max _{\mathbf{x} \in \Omega} u(\mathbf{x}) \geq 0$. By (2.23), we have

$$
\begin{aligned}
\mathcal{L} u\left(\mathbf{x}_{0}\right)= & -\int_{\Omega}\left(a\left(\mathbf{x}_{0}\right)+a(\mathbf{y})\right)\left(u(\mathbf{y})-u\left(\mathbf{x}_{0}\right)\right) 1_{d}\left(\mathbf{x}_{0}, \mathbf{y}\right) \gamma_{d}\left(\left\|\mathbf{y}-\mathbf{x}_{0}\right\|\right) d \mathbf{y} \\
& +\int_{\Omega}\left(u(\mathbf{y})-u\left(\mathbf{x}_{0}\right)\right) \boldsymbol{b}(\mathbf{y})^{T} \frac{\mathbf{y}-\mathbf{x}_{0}}{\left\|\mathbf{y}-\mathbf{x}_{0}\right\|} 1_{c}\left(\mathbf{y}, \mathbf{x}_{0}\right) \gamma_{c}\left(\left\|\mathbf{y}-\mathbf{x}_{0}\right\|\right) d \mathbf{y} \\
& +u\left(\mathbf{x}_{0}\right) \mathcal{D}(\boldsymbol{\mu})\left(\mathbf{x}_{0}\right) .
\end{aligned}
$$

Since $u(\mathbf{y})-u\left(\mathbf{x}_{0}\right) \leq 0$, it is easy to verify that the first integral in (2.24) satisfies

$$
-\int_{\Omega} \overbrace{\left(a\left(\mathbf{x}_{0}\right)+a(\mathbf{y})\right)}^{>0} \overbrace{\left(u(\mathbf{y})-u\left(\mathbf{x}_{0}\right)\right)}^{\leq 0} \overbrace{1_{d}\left(\mathbf{x}_{0}, \mathbf{y}\right) \gamma_{d}\left(\left\|\mathbf{y}-\mathbf{x}_{0}\right\|\right)}^{\geq 0} d \mathbf{y} \geq 0 .
$$

According to the definition of $1_{c}\left(\mathbf{y}, \mathbf{x}_{0}\right)$, the second integral of $(2.24)$ gives us

$$
\int_{\Omega} \overbrace{\left(u(\mathbf{y})-u\left(\mathbf{x}_{0}\right)\right)}^{\leq 0} \overbrace{\boldsymbol{b}(\mathbf{y})^{T} \frac{\mathbf{y}-\mathbf{x}_{0}}{\left\|\mathbf{y}-\mathbf{x}_{0}\right\|} 1_{c}\left(\mathbf{y}, \mathbf{x}_{0}\right)}^{\leq 0} \overbrace{\gamma_{c}\left(\left\|\mathbf{y}-\mathbf{x}_{0}\right\|\right)}^{\geq 0} d \mathbf{y} \geq 0 .
$$

By the given condition that $D(\boldsymbol{\mu})(\mathbf{x}) \geq 0$ for $\mathbf{x} \in \Omega_{s}$ and $u\left(\mathbf{x}_{0}\right) \geq 0$, it holds that $u\left(\mathbf{x}_{0}\right) \mathcal{D}(\boldsymbol{\mu})\left(\mathbf{x}_{0}\right) \geq$ 0 . Hence, we get $\mathcal{L} u\left(\mathbf{x}_{0}\right) \geq 0$, which is a contradiction with the assumption of $\mathcal{L} u\left(\mathbf{x}_{0}\right)<0$. 
Remark 2. As for the condition $\mathcal{D}(\boldsymbol{\mu})(\mathbf{x}) \geq 0$ for $\mathbf{x} \in \Omega_{s}$, we observe that

- if $\boldsymbol{b}(\mathbf{x})$ is a constant vector, then $\mathcal{D}(\boldsymbol{\mu})(\mathbf{x})=0$;

- if $\nabla \cdot \boldsymbol{b}$ is continuous and strictly positive in $\Omega$, then $\mathcal{D}(\boldsymbol{\mu})(\mathbf{x})>0$ for all $\mathbf{x} \in \Omega_{s}$ when $\delta>0$ is sufficiently small.

Moreover, we note that while the nonlocal diffusion-convection model proposed in [7] is conservative, it does not satisfy the maximum principle in general due to the loss of the upwind feature.

\subsection{Weak form and well-posedness}

The equation (2.14) can be regarded as the strong form of our nonlocal convection-diffusion model. Without loss of generality, we here consider the case of homogeneous nonlocal boundary condition $g(\mathbf{x})=0$ for $\mathbf{x} \in \Omega_{c}$ and $f \in L^{2}\left(\Omega_{s}\right)$. Let us define the constrained space $L_{n 0}^{2}(\Omega)=\{u \in$ $L^{2}(\Omega): u(\mathbf{x})=0$ on $\left.\Omega_{c}\right\}$ and denote by $(\cdot, \cdot)$ the $L^{2}$ inner product. Then a weak formulation of the nonlocal convection-diffusion problem $(2.14)$ is to find $u \in L_{n 0}^{2}(\Omega)$ such that

$$
\mathbf{B}(u, v)=\mathbf{F}(v), \quad \forall v \in L_{n 0}^{2}(\Omega),
$$

where the bilinear functional $\mathbf{B}(u, v)=(\mathcal{L} u, v)$ and the linear functional $\mathbf{F}(v)=(f, v)$. Let us consider the weight functions $\omega_{d}$ and $\omega_{c}$ in (2.4) and (2.10), such as those determined by $\gamma_{d}$ and $\gamma_{c}$ given in (2.3) and (2.9) with $0 \leq s_{d}, s_{c}<n$. Furthermore, with $0<a_{0} \leq a(\mathbf{x}) \leq a_{1}<\infty$ and $0 \leq \boldsymbol{b}(\mathbf{x})^{T}(\mathbf{y}-\mathbf{x}) 1_{c}(\mathbf{x}, \mathbf{y}) \leq \hat{b} \operatorname{diam}(\Omega)<\infty$ for any $\mathbf{x}, \mathbf{y} \in \Omega$, it is not hard to find that the kernel $\gamma$ satisfies

$$
\int_{\Omega} \gamma(\mathbf{x}, \mathbf{y}) d \mathbf{y} \leq K_{1}(\delta), \quad \int_{\Omega} \gamma(\mathbf{y}, \mathbf{x}) d \mathbf{y} \leq K_{1}(\delta), \quad \forall \mathbf{x} \in \Omega
$$

and

$$
\int_{\Omega_{c}} \gamma(\mathbf{y}, \mathbf{x}) d \mathbf{y} \geq K_{2}(\delta), \quad \forall \mathbf{x} \in \Omega
$$

for some positive constants $K_{1}(\delta)$ and $K_{2}(\delta)$. Under these conditions, the nonlocal operator $\mathcal{L}$ is bounded in $L_{n 0}^{2}(\Omega)$ so that a weak solution is also a strong solution of $(2.13)$ in $L_{n 0}^{2}(\Omega)$. The well-posedness of the nonlocal convection-diffusion model proposed in [7] has been proved for a symmetric convection kernel $\boldsymbol{\mu}(\mathbf{x}, \mathbf{y})$. We modify the techniques used in [7] to obtain the following result for our nonlocal model having no symmetry in the convection kernel.

Theorem 2. (Well-posedness) Let $\delta>0$ be fixed and assume

$$
K_{2}(\delta)+\min _{\mathbf{x} \in \Omega_{x}} \mathcal{D}(\boldsymbol{\mu})(\mathbf{x})=: C_{\text {coer }}>0 .
$$

Then there exists a unique solution $u \in L_{n 0}^{2}(\Omega)$ to the nonlocal convection-diffusion problem (2.13). Furthermore, the solution satisfies the a priori estimate

$$
\|u\|_{L^{2}(\Omega)} \leq \frac{2}{C_{\text {coer }}}\|f\|_{L^{2}(\Omega)} .
$$


Proof. First, it is easy to see that

$$
|\mathbf{F}(v)| \leq\|f\|_{L^{2}(\Omega)}\|v\|_{L^{2}\left(\Omega_{s}\right)} .
$$

We now show that the bilinear operator $\mathbf{B}(\cdot, \cdot)$ is bounded on $L_{n 0}^{2}(\Omega) \times L_{n 0}^{2}(\Omega)$. It holds that for any $u, v \in L_{n 0}^{2}(\Omega)$,

$$
\begin{aligned}
\mathbf{B}(u, v)= & \int_{\Omega} \int_{\Omega}(u(\mathbf{x}) \boldsymbol{\gamma}(\mathbf{x}, \mathbf{y})-u(\mathbf{y}) \boldsymbol{\gamma}(\mathbf{y}, \mathbf{x})) v(\mathbf{x}) d \mathbf{y} d \mathbf{x} \\
= & \frac{1}{2} \int_{\Omega} \int_{\Omega} u(\mathbf{y}) v(\mathbf{y}) \boldsymbol{\gamma}(\mathbf{y}, \mathbf{x})+u(\mathbf{x}) v(\mathbf{x}) \boldsymbol{\gamma}(\mathbf{x}, \mathbf{y}) d \mathbf{y} d \mathbf{x} \\
& -\frac{1}{2} \int_{\Omega} \int_{\Omega} u(\mathbf{y}) v(\mathbf{x}) \boldsymbol{\gamma}(\mathbf{y}, \mathbf{x})+u(\mathbf{x}) v(\mathbf{y}) \boldsymbol{\gamma}(\mathbf{x}, \mathbf{y}) d \mathbf{y} d \mathbf{x} \\
= & \int_{\Omega} \int_{\Omega} u(\mathbf{x}) v(\mathbf{x}) \boldsymbol{\gamma}(\mathbf{x}, \mathbf{y}) d \mathbf{y} d \mathbf{x}-\int_{\Omega} \int_{\Omega} u(\mathbf{x}) v(\mathbf{y}) \boldsymbol{\gamma}(\mathbf{x}, \mathbf{y}) d \mathbf{y} d \mathbf{x}
\end{aligned}
$$

Using the inequality in (2.26), we have

$$
\begin{aligned}
\left|\int_{\Omega} \int_{\Omega} u(\mathbf{x}) v(\mathbf{x}) \boldsymbol{\gamma}(\mathbf{x}, \mathbf{y}) d \mathbf{y} d \mathbf{x}\right| & =\left|\int_{\Omega} u(\mathbf{x}) v(\mathbf{x}) \int_{\Omega} \gamma(\mathbf{x}, \mathbf{y}) d \mathbf{y} d \mathbf{x}\right| \\
& \leq K_{1}(\delta)\|u\|_{L^{2}(\Omega)}\|v\|_{L^{2}(\Omega)}
\end{aligned}
$$

and

$$
\begin{aligned}
& \left|\int_{\Omega} \int_{\Omega}(u(\mathbf{x}) v(\mathbf{y}) \boldsymbol{\gamma}(\mathbf{x}, \mathbf{y})) d \mathbf{y} d \mathbf{x}\right| \\
& \quad \leq\left(\int_{\Omega} \int_{\Omega} u^{2}(\mathbf{x}) \gamma(\mathbf{x}, \mathbf{y}) d \mathbf{y} d \mathbf{x}\right)^{1 / 2}\left(\int_{\Omega} \int_{\Omega} v(\mathbf{y})^{2} \gamma(\mathbf{x}, \mathbf{y}) d \mathbf{y} d \mathbf{x}\right)^{1 / 2} \\
& \quad=\left(\int_{\Omega} u^{2}(\mathbf{x}) \int_{\Omega} \gamma(\mathbf{x}, \mathbf{y}) d \mathbf{y} d \mathbf{x}\right)^{1 / 2}\left(\int_{\Omega} v(\mathbf{x})^{2} \int_{\Omega} \gamma(\mathbf{y}, \mathbf{x}) d \mathbf{y} d \mathbf{x}\right)^{1 / 2} \\
& \quad \leq K_{1}(\delta)\|u\|_{L^{2}(\Omega)}\|v\|_{L^{2}(\Omega)},
\end{aligned}
$$

so that

$$
|\mathbf{B}(u, v)| \leq 2 K_{1}(\delta)\|u\|_{L^{2}(\Omega)}\|v\|_{L^{2}(\Omega)} .
$$

Next we show that the bilinear operator $B(\cdot, \cdot)$ is coercive on $L_{n 0}^{2}(\Omega)$. We first note that

$$
\begin{aligned}
\mathbf{B}(u, u)= & \int_{\Omega} \int_{\Omega}(u(\mathbf{x}) \gamma(\mathbf{x}, \mathbf{y})-u(\mathbf{y}) \gamma(\mathbf{y}, \mathbf{x})) u(\mathbf{x}) d \mathbf{y} d \mathbf{x} \\
= & \frac{1}{2} \int_{\Omega} \int_{\Omega}(u(\mathbf{y}) \gamma(\mathbf{y}, \mathbf{x})-u(\mathbf{x}) \gamma(\mathbf{x}, \mathbf{y}))(u(\mathbf{y})-u(\mathbf{x})) d \mathbf{y} d \mathbf{x} \\
= & \frac{1}{2} \int_{\Omega} \int_{\Omega}(u(\mathbf{y})-u(\mathbf{x}))^{2} \gamma(\mathbf{y}, \mathbf{x}) d \mathbf{y} d \mathbf{x} \\
& +\frac{1}{2} \int_{\Omega} \int_{\Omega} u(\mathbf{x})(u(\mathbf{y})-u(\mathbf{x}))(\gamma(\mathbf{y}, \mathbf{x})-\gamma(\mathbf{x}, \mathbf{y})) d \mathbf{y} d \mathbf{x}
\end{aligned}
$$


For the first term on the right hand side of in (2.34), we have

$$
\begin{aligned}
\frac{1}{2} \int_{\Omega} \int_{\Omega} & (u(\mathbf{y})-u(\mathbf{x}))^{2} \gamma(\mathbf{y}, \mathbf{x}) d \mathbf{y} d \mathbf{x} \\
\geq & \frac{1}{2} \int_{\Omega} \int_{\Omega_{c}}(u(\mathbf{y})-u(\mathbf{x}))^{2} \gamma(\mathbf{y}, \mathbf{x}) d \mathbf{y} d \mathbf{x} \\
\quad= & \frac{1}{2} \int_{\Omega} u(\mathbf{x})^{2} \int_{\Omega_{c}} \gamma(\mathbf{y}, \mathbf{x}) d \mathbf{y} d \mathbf{x} \geq \frac{K_{2}(\delta)}{2}\|u\|_{L^{2}(\Omega)}^{2} .
\end{aligned}
$$

For the second term, we have

$$
\begin{aligned}
\frac{1}{2} \int_{\Omega} \int_{\Omega} & u(\mathbf{x})(u(\mathbf{y})-u(\mathbf{x}))(\gamma(\mathbf{y}, \mathbf{x})-\gamma(\mathbf{x}, \mathbf{y})) d \mathbf{y} d \mathbf{x} \\
= & \frac{1}{2} \int_{\Omega} \int_{\Omega} u^{2}(\mathbf{x})(\gamma(\mathbf{x}, \mathbf{y})-\gamma(\mathbf{y}, \mathbf{x})) d \mathbf{y} d \mathbf{x} \\
& =\frac{1}{2} \int_{\Omega} u^{2}(\mathbf{x}) \int_{\Omega}\left(\boldsymbol{b}(\mathbf{x}) \omega_{c}(\mathbf{x}, \mathbf{y})+\boldsymbol{b}(\mathbf{y}) \omega_{c}(\mathbf{y}, \mathbf{x})\right)^{T} \frac{\mathbf{y}-\mathbf{x}}{\|\mathbf{y}-\mathbf{x}\|} d \mathbf{y} d \mathbf{x} \\
& =\frac{1}{2} \int_{\Omega_{s}} u^{2}(\mathbf{x}) \mathcal{D}(\boldsymbol{\mu})(\mathbf{x}) d \mathbf{x} .
\end{aligned}
$$

So we obtain from (2.35) and (2.36) that

$$
\mathbf{B}(u, u) \geq \frac{1}{2} \int_{\Omega_{s}} u^{2}(\mathbf{x})\left(K_{2}(\delta)+\mathcal{D}(\boldsymbol{\mu})(\mathbf{x})\right) d \mathbf{x} \geq \frac{C_{c o e r}}{2}\|u\|_{L^{2}(\Omega)}^{2} .
$$

Therefore, there exists a unique weak (and thus strong) solution $u \in L_{n 0}^{2}(\Omega)$ to the problem (2.25) by the Lax-Milgram theorem. Finally, because

$$
\frac{C_{\mathrm{coer}}}{2}\|u\|_{L^{2}(\Omega)}^{2} \leq \boldsymbol{B}(u, u)=|\mathbf{F}(u)| \leq\|f\|_{L^{2}(\Omega)}\|u\|_{L^{2}(\Omega)},
$$

we have the a priori estimate (2.28).

\subsection{Local limit}

Next we derive the local limit of the nonlocal weak formulation $(2.25)$ as the horizon $\delta \rightarrow 0$. For any $u, v \in L^{2}(\Omega)$, let $(u, v)$ denote their $L^{2}$ inner product.

Theorem 3. (Local limit) If $u, v \in L_{n 0}^{2}(\Omega) \cap C^{2}(\bar{\Omega})$, then

$$
\lim _{\delta \rightarrow 0} \mathbf{B}(u, v)=(a \nabla u, \nabla v)-(\boldsymbol{b} u, \nabla v) .
$$

Proof. Note that

$$
\begin{aligned}
\mathbf{B}(u, v) & =\int_{\Omega} \int_{\Omega}(u(\mathbf{x}) \boldsymbol{\gamma}(\mathbf{x}, \mathbf{y})-u(\mathbf{y}) \boldsymbol{\gamma}(\mathbf{y}, \mathbf{x})) v(\mathbf{x}) d \mathbf{y} d \mathbf{x} \\
& =\int_{\Omega} \int_{\Omega}(u(\mathbf{y}) \boldsymbol{\gamma}(\mathbf{y}, \mathbf{x})-u(\mathbf{x}) \boldsymbol{\gamma}(\mathbf{x}, \mathbf{y})) v(\mathbf{y}) d \mathbf{y} d \mathbf{x} \\
& =\frac{1}{2} \int_{\Omega} \int_{\Omega}(u(\mathbf{y}) \boldsymbol{\gamma}(\mathbf{y}, \mathbf{x})-u(\mathbf{x}) \boldsymbol{\gamma}(\mathbf{x}, \mathbf{y}))(v(\mathbf{y})-v(\mathbf{x})) d \mathbf{y} d \mathbf{x}
\end{aligned}
$$




$$
\begin{aligned}
= & \frac{1}{2} \int_{\Omega} \int_{\Omega}(a(\mathbf{x})+a(\mathbf{y}))(u(\mathbf{y})-u(\mathbf{x})(v(\mathbf{y})-v(\mathbf{x}))) \omega_{d}(\mathbf{x}, \mathbf{y}) d \mathbf{y} d \mathbf{x}-\frac{1}{2} \int_{\Omega} \int_{\Omega} \\
& \left(\boldsymbol{b}(\mathbf{x}) u(\mathbf{x}) \omega_{c}(\mathbf{x}, \mathbf{y})+\boldsymbol{b}(\mathbf{y}) u(\mathbf{y}) \omega_{c}(\mathbf{y}, \mathbf{x})\right)^{T} \frac{\mathbf{y}-\mathbf{x}}{\|\mathbf{y}-\mathbf{x}\|}(v(\mathbf{y})-v(\mathbf{x})) d \mathbf{y} d \mathbf{x} \\
= & \boldsymbol{B}^{\operatorname{diff}}(u, v)+\boldsymbol{B}^{\operatorname{conv}}(u, v),
\end{aligned}
$$

where $B^{\text {diff }}(u, v)$ denotes the nonlocal diffusion contribution and $B^{\text {conv }}(u, v)$ the nonlocal convection contribution. Using Taylor's expansion of $u$ at $\mathbf{x}$ and interchanging the variables $\mathbf{x}$ and $\mathbf{y}$, the nonlocal diffusion part in (2.40) can be written as

$$
\begin{aligned}
\boldsymbol{B}^{\text {diff }}(u, v) & =\int_{\Omega} a(\mathbf{x}) \int_{\Omega}(u(\mathbf{y})-u(\mathbf{x}))(v(\mathbf{y})-v(\mathbf{x})) \omega_{d}(\mathbf{x}, \mathbf{y}) d \mathbf{y} d \mathbf{x} \\
& =\int_{\Omega} a(\mathbf{x}) \int_{B(\mathbf{0}, \delta)}\left(\nabla u(\mathbf{x})^{T} \mathbf{z z}^{T} \nabla v(\mathbf{x})+o\left(\|\mathbf{z}\|^{2}\right)\right) \gamma_{d}(\|\mathbf{z}\|) d \mathbf{z} d \mathbf{x} .
\end{aligned}
$$

According to (2.2) and(2.41), we obtain by simple calculations that

$$
\lim _{\delta \rightarrow 0} \boldsymbol{B}^{\mathrm{diff}}(u, v)=\lim _{\delta \rightarrow 0}\left(\int_{\Omega} a(\mathbf{x}) \nabla u(\mathbf{x})^{T} \nabla v(\mathbf{x}) d \mathbf{x}+o(1)\right)=(a \nabla u, \nabla v) .
$$

For the nonlocal convection part in (2.40), we similarly have

$$
\begin{aligned}
\boldsymbol{B}^{\mathrm{conv}}(u, v) & =-\int_{\Omega} \int_{\Omega}(\boldsymbol{b}(\mathbf{x}) u(\mathbf{x}))^{T} \frac{\mathbf{y}-\mathbf{x}}{\|\mathbf{y}-\mathbf{x}\|}(v(\mathbf{y})-v(\mathbf{x})) \omega_{c}(\mathbf{x}, \mathbf{y}) d \mathbf{y} d \mathbf{x} \\
& =-\int_{\Omega} \int_{\widetilde{H B}(\mathbf{x}, \delta)}\left(\boldsymbol{b}^{T}(\mathbf{x}) u(\mathbf{x}) \frac{(\mathbf{y}-\mathbf{x})(\mathbf{y}-\mathbf{x})^{T}}{\|\mathbf{y}-\mathbf{x}\|} \nabla v(\mathbf{x})+o(\|\mathbf{y}-\mathbf{x}\|)\right) \\
& \gamma_{c}(\|\mathbf{y}-\mathbf{x}\|) d \mathbf{y} d \mathbf{x} \\
& -\int_{\Omega} \int_{B(\mathbf{0}, \delta)}\left(\boldsymbol{b}^{T}(\mathbf{x}) u(\mathbf{x}) \frac{\mathbf{z z} \mathbf{z}^{T}}{2\|\mathbf{z}\|} \nabla v(\mathbf{x})+o(\|\mathbf{z}\|)\right) \gamma_{c}(\|\mathbf{z}\|) d \mathbf{z} d \mathbf{x}
\end{aligned}
$$

where in the last step, the symmetry of the integral with respect to $z_{i} z_{j} /\|\mathbf{z}\|$ has been used. According to (2.8) and (2.43), we then get

$$
\lim _{\delta \rightarrow 0} \boldsymbol{B}^{\mathrm{conv}}(u, v)=\lim _{\delta \rightarrow 0}\left(\int_{\Omega} \boldsymbol{b}(\mathbf{x})^{T} u(\mathbf{x}) \nabla v(\mathbf{x}) d \mathbf{x}+o(1)\right)=-(\boldsymbol{b} u, \nabla v) .
$$

Finally, a combination of (2.42) and (2.44) gives us (2.39).

Note that the right hand side of (2.39) is exactly the bilinear operator associated with the local convection-diffusion problem (2.15) with $g=0$.

\section{A quadrature-based finite difference discretization and its analysis}

In this section we propose and analyze a quadrature-based finite difference scheme for discretizing the nonlocal convection-diffusion problem (2.13). 


\subsection{Discretization scheme}

Let us denote the nodes of a rectangular grid $\mathcal{T}_{h}$ of $\Omega$ as $\left\{\mathbf{x}_{1}, \mathbf{x}_{2}, \cdots, \mathbf{x}_{N_{s}}\right\} \in \Omega_{s}$ (interior nodes) and $\left\{\mathbf{x}_{N_{s}+1}, \mathbf{x}_{N_{s}+2}, \cdots, \mathbf{x}_{N_{s}+K_{c}}\right\} \in \Omega_{c}$ (nonlocal boundary nodes), and denote by $\phi_{i}(\mathbf{x})$ the standard continuous piecewise bilinear (if $n=2$ ) or trilinear (if $n=3$ ) basis function at the point $\mathbf{x}_{i}$ and by $S_{i}$ the support region for each $\phi_{i}(\mathbf{x})$. We also assume that the grid is uniform in all directions with the same grid size $h$ and thus locally symmetric in all directions at each interior nodes. For the diffusion term in (2.14), we follow [41] and its multidimensional generalization [16] but with some necessary modifications to incorporate the inhomogeneous coefficient $a=a(\mathbf{x})$. At the node $\mathbf{x}_{i}$ for $i=1,2, \cdots, N_{s}$,

$$
\mathcal{L}^{\text {diff }} u\left(\mathbf{x}_{i}\right)=-\int_{B\left(\mathbf{x}_{i}, \delta\right)}\left(a(\mathbf{y})+a\left(\mathbf{x}_{i}\right)\right)\left(u(\mathbf{y})-u\left(\mathbf{x}_{i}\right)\right) \gamma_{d}\left(\left\|\mathbf{y}-\mathbf{x}_{i}\right\|\right) d \mathbf{y}
$$

then we approximate it by

$$
\mathcal{L}_{h}^{\operatorname{diff}} u\left(\mathbf{x}_{i}\right)=-\int_{B\left(\mathbf{x}_{i}, \delta\right)} \mathcal{I}_{h}\left(\frac{\left(a(\mathbf{y})+a\left(\mathbf{x}_{i}\right)\right)\left(u(\mathbf{y})-u\left(\mathbf{x}_{i}\right)\right)}{G\left(\mathbf{y}-\mathbf{x}_{i}\right)}\right) G\left(\mathbf{y}-\mathbf{x}_{i}\right) \gamma_{d}\left(\left\|\mathbf{y}-\mathbf{x}_{i}\right\|\right) d \mathbf{z},
$$

where $\mathcal{I}_{h}(\cdot)$ represents the piecewise bilinear (or trilinear) interpolation operator associated with the grid $\mathcal{T}_{h}$, and $G(\mathbf{z})$ represents a weight function defined by

$$
G(\mathbf{z})=\frac{\|\mathbf{z}\|^{2}}{\left|z_{1}\right|+\cdots+\left|z_{n}\right|} .
$$

The introduction of the term $G=G(\mathbf{z})$ has been first proposed in [16]. It is an important ingredient when extending the scheme constructed in [41] to the multidimensional case. Such a discretization leads to the so-called asymptotic compatibility (see [42] for a precise definition) for the constant diffusion coefficient case as demonstrated in [16]. Further discussions are given later in this work as well. Clearly (3.2) can be further rewritten as

$$
\begin{aligned}
\mathcal{L}_{h}^{\text {diff }} u\left(\mathbf{x}_{i}\right)=-\sum_{j \neq i}\left(u\left(\mathbf{x}_{j}\right)-u\left(\mathbf{x}_{i}\right)\right) \frac{a\left(\mathbf{x}_{j}\right)+a\left(\mathbf{x}_{i}\right)}{G\left(\mathbf{x}_{j}-\mathbf{x}_{i}\right)} \\
\int_{B\left(\mathbf{x}_{i}, \delta\right)} \phi_{j}(\mathbf{y}) G\left(\mathbf{y}-\mathbf{x}_{i}\right) \gamma_{d}\left(\left\|\mathbf{y}-\mathbf{x}_{i}\right\|\right) d \mathbf{y} .
\end{aligned}
$$

For the convection term (2.14), we have at $\mathbf{x}_{i}$ that

$$
\begin{aligned}
\mathcal{L}^{\text {conv }} u\left(\mathbf{x}_{i}\right)= & \int_{B\left(\mathbf{x}_{i}, \delta\right)}\left(u(\mathbf{y})-u\left(\mathbf{x}_{i}\right)\right) \boldsymbol{b}(\mathbf{y})^{T} \frac{\mathbf{y}-\mathbf{x}_{i}}{\left\|\mathbf{y}-\mathbf{x}_{i}\right\|} 1_{c}\left(\mathbf{y}, \mathbf{x}_{i}\right) \gamma_{c}\left(\left\|\mathbf{y}-\mathbf{x}_{i}\right\|\right) d \mathbf{y} \\
& +u\left(\mathbf{x}_{i}\right) \mathcal{D}(\boldsymbol{\mu})\left(\mathbf{x}_{i}\right),
\end{aligned}
$$

then we approximate it by

$$
\begin{aligned}
\mathcal{L}_{h}^{\text {conv }} u\left(\mathbf{x}_{i}\right)= & \int_{B\left(\mathbf{x}_{i}, \delta\right)} \mathcal{I}_{h}\left(u(\mathbf{y})-u\left(\mathbf{x}_{i}\right)\right) \boldsymbol{b}(\mathbf{y})^{T} \frac{\mathbf{y}-\mathbf{x}_{i}}{\left\|\mathbf{y}-\mathbf{x}_{i}\right\|} 1_{c}\left(\mathbf{y}, \mathbf{x}_{i}\right) \gamma_{c}\left(\left\|\mathbf{y}-\mathbf{x}_{i}\right\|\right) d \mathbf{y} \\
& +u\left(\mathbf{x}_{i}\right) \mathcal{D}(\boldsymbol{\mu})\left(\mathbf{x}_{i}\right)
\end{aligned}
$$


which is equivalent to

$$
\begin{aligned}
\mathcal{L}_{h}^{\text {conv }} u\left(\mathbf{x}_{i}\right)= & \sum_{j \neq i}\left(u\left(\mathbf{x}_{j}\right)-u\left(\mathbf{x}_{i}\right)\right) \int_{B\left(\mathbf{x}_{i}, \delta\right)} \phi_{j}(\mathbf{y}) \boldsymbol{b}(\mathbf{y})^{T} \frac{\mathbf{y}-\mathbf{x}_{i}}{\left\|\mathbf{y}-\mathbf{x}_{i}\right\|} 1_{c}\left(\mathbf{y}, \mathbf{x}_{i}\right) \gamma_{c}\left(\left\|\mathbf{y}-\mathbf{x}_{i}\right\|\right) d \mathbf{y} \\
& +u\left(\mathbf{x}_{i}\right) \mathcal{D}(\boldsymbol{\mu})\left(\mathbf{x}_{i}\right)
\end{aligned}
$$

for $i=1,2, \cdots, N_{s}$.

Finally, a quadrature-based finite difference scheme for solving the nonlocal convection-diffusion problem (2.13) can be given as follows: find $\left(u_{h}\left(\mathbf{x}_{1}\right), \cdots, u_{h}\left(\mathbf{x}_{N_{s}}\right)\right)$ such that

$$
\mathcal{L}_{h} u_{h}\left(\mathbf{x}_{i}\right)=f\left(\mathbf{x}_{i}\right), \quad i=1, \cdots, N_{s},
$$

where $\mathcal{L}_{h} u_{h}\left(\mathbf{x}_{i}\right)=\mathcal{L}_{h}^{\text {diff }} u_{h}\left(\mathbf{x}_{i}\right)+\mathcal{L}_{h}^{\text {conv }} u_{h}\left(\mathbf{x}_{i}\right)$.

\subsection{Stability and consistency}

Let us define

$$
\begin{aligned}
q_{i, j}^{d} & =\frac{a\left(\mathbf{x}_{j}\right)+a\left(\mathbf{x}_{i}\right)}{G\left(\mathbf{x}_{j}-\mathbf{x}_{i}\right)} \int_{B\left(\mathbf{x}_{i}, \delta\right)} G\left(\mathbf{y}-\mathbf{x}_{i}\right) \phi_{j}(\mathbf{y}) \gamma_{d}\left(\left\|\mathbf{y}-\mathbf{x}_{i}\right\|\right) d \mathbf{y}, \\
q_{i, j}^{c} & =\int_{B\left(\mathbf{x}_{i}, \delta\right)} \phi_{j}(\mathbf{y}) \boldsymbol{b}(\mathbf{y})^{T} \frac{\mathbf{y}-\mathbf{x}_{i}}{\left\|\mathbf{y}-\mathbf{x}_{i}\right\|} 1_{c}\left(\mathbf{y}, \mathbf{x}_{i}\right) \gamma_{c}\left(\left\|\mathbf{y}-\mathbf{x}_{i}\right\|\right) d \mathbf{y}
\end{aligned}
$$

for $j \neq i$. It is easy to show that $q_{i, j}^{d}$ and $q_{i, j}^{c}$ are all finite for any given $\delta>0$. Furthermore $q_{i, j}^{d}=0$ if $B\left(\mathbf{x}_{i}, \delta\right) \cap S_{j}=\emptyset$ and $q_{i, j}^{c}=0$ if $H B\left(\mathbf{x}_{i}, \delta\right) \cap S_{j}=\emptyset$ since $\phi_{j}(\mathbf{y})=0$ for $\mathbf{y} \notin S_{j}$. Then the resulting linear system from the finite difference discretization (3.7) can be expressed by

$$
A_{h} \vec{u}_{h}=\vec{f}
$$

where $\vec{u}_{h}=\left(u_{h}\left(\mathbf{x}_{1}\right), \cdots, u_{h}\left(\mathbf{x}_{N_{s}}\right)\right)^{T}, \vec{f}=\left(f_{1}, \cdots, f_{N_{s}}\right)^{T}$ with

$$
f_{i}=f\left(\mathbf{x}_{i}\right)+\sum_{j=N_{s}+1}^{N_{s}+K_{c}} u\left(\mathbf{x}_{j}\right)\left(q_{i, j}^{d}-q_{i, j}^{c}\right),
$$

and $A_{h}=\left(a_{i, j}\right)_{N_{s} \times N_{s}}$ with

$$
a_{i, j}= \begin{cases}-q_{i, j}^{d}+q_{i, j}^{c}, & \text { if } j \neq i \\ \sum_{k \neq i}\left[q_{i, k}^{d}-q_{i, k}^{c}\right]+\mathcal{D}(\boldsymbol{\mu})\left(\mathbf{x}_{i}\right), & \text { if } j=i\end{cases}
$$

for $1 \leq i, j \leq N_{s}$.

Theorem 4. Let the horizon $\delta$ be fixed and suppose that $\mathcal{D}(\boldsymbol{\mu}) \geq 0$ in $\Omega_{s}$, then the stiffness matrix $A_{h}$ defined in (3.10) is a nonsingular M-matrix ${ }^{1}$. Consequently, the linear system (3.8) corresponding to the quadrature-based finite difference discretization (3.7) of the nonlocal convection-diffusion problem (2.13) is uniquely solvable, and $u_{h}$ always satisfies the discrete maximum principle: if $f \leq 0$ in $\Omega_{s}$, then $\max _{1 \leq i \leq N} u_{h}\left(\mathbf{x}_{i}\right) \leq 0$, and if $f \geq 0$ in $\Omega_{s}$, then $\min _{1 \leq i \leq N} u_{h}\left(\mathbf{x}_{i}\right) \geq 0$.

\footnotetext{
${ }^{1} \mathrm{~A}$ matrix is a nonsingular $M$-matrix if it has positive diagonal entries and nonpositive off-diagonal entries and is strictly diagonally dominant. A nonsingular $M$-matrix is inverse-positive and the real part of each of its eigenvalues is positive [32].
} 
Proof. First it is clear that $q_{i, j}^{d}>0$ if $i \neq j$ and $B\left(\mathbf{x}_{i}, \delta\right) \cap S_{j} \neq \emptyset$ since $G\left(\mathbf{x}_{j}-\mathbf{x}_{i}\right)>0$ and $a\left(\mathbf{x}_{i}\right)+a\left(\mathbf{x}_{j}\right) \geq 2 a_{0}>0$. For any $\mathbf{y} \in H B\left(\mathbf{x}_{i}, \delta\right)$, since $\boldsymbol{b}(\mathbf{y})^{T}\left(\mathbf{x}_{i}-\mathbf{y}\right) \geq 0$, we get for any $i \neq j$ that $q_{i, j}^{c} \leq 0$ if $H B\left(\mathbf{x}_{i}, \delta\right) \cap S_{j} \neq \emptyset$. Thus by (3.10), it holds that for any $j \neq i$, we have $a_{i, j}<0$ if $B(\mathbf{x}, \delta) \cap S_{j} \neq \emptyset$ and $a_{i, j}=0$ if $B\left(\mathbf{x}_{i}, \delta\right) \cap S_{j}=\emptyset$. Consequently we have $a_{i, i}>0$ since $D(\boldsymbol{\mu})\left(\mathbf{x}_{i}\right) \geq 0$. It is also easy to check that if $S_{i} \cap \Omega_{c}=\emptyset$ then $\sum_{j=1}^{N_{s}} a_{i, j}=\mathcal{D}(\boldsymbol{\mu})\left(\mathbf{x}_{i}\right) \geq 0$; otherwise $\sum_{j=1}^{N_{s}} a_{i, j}>0$. Thus, the stiffness matrix $A_{h}$ is an $M$-matrix, which means that $A_{h}^{-1}$ exists and does not have negative entries. The unique solvability of the linear system (3.8) and the discrete maximum principle then directly follows.

Theorem 4 guarantees the numerical stability of the proposed quadrature-based finite difference discretization (3.7). By using the linear interpolation property and analysis similar to that in [41], we can obtain the following estimate on the consistency (truncation) error of (3.7) under additional smoothness assumptions on the exact solutions of nonlocal models.

Theorem 5. Let the horizon $\delta>0$ be fixed and assume that the solution of the nonlocal convectiondiffusion problem (2.13) $u \in C^{3}(\bar{\Omega})$, then the quadrature-based finite difference discretization (3.7) satisfies

$$
\max _{1 \leq i \leq N_{s}}\left|\mathcal{L}_{h} u\left(\mathbf{x}_{i}\right)-\mathcal{L} u\left(\mathbf{x}_{i}\right)\right|=O\left(h^{2}\right)
$$

for $h>0$ sufficiently small.

Remark 3. Theorem 5 further tells us that when the horizon $\delta>0$ is fixed, we expect to observe second-order convergence, with respect to the grid size $h$, of the approximate solutions to the exact solution of the nonlocal problem (2.13) as $h \rightarrow 0$, at least for smooth exact solutions.

\subsection{Asymptotic compatibility}

In practical simulations of nonlocal models, the grid size $h$ is sometimes coupled together with the horizon $\delta$, see [36]. We are concerned with the asymptotic compatibility of the proposed quadrature-based finite difference scheme (3.7), i.e., the convergence behavior of the approximate solutions to the exact solution of the corresponding local convection-diffusion problem (2.15) when $\delta \rightarrow 0$ and $h \rightarrow 0$ simultaneously occur. In particular, the convergence under the condition of $\delta / h=O(1)$ has been called " $\delta$-convergence" in the numerical study of nonlocal peridynamics [3]. We adopt this convention for convenience in making reference.

First let us consider the approximate nonlocal diffusion term at each node $\mathbf{x}_{i}$ defined by (3.2). A special case has been derived in [16] for the nonlocal diffusion model with a constant coefficient (describing a homogeneous materials). Our result here extends to the variable coefficient case $a=a(\mathbf{x})$ and the detailed derivations are given below for completeness. Again, we note that additional smoothness assumptions on the exact solution are made.

Theorem 6. Assume that $u \in C^{4}(\bar{\Omega}), a \in C^{3}(\bar{\Omega})$, and let $\mathcal{L}_{h}^{\text {diff }} u\left(\mathbf{x}_{i}\right)=-\nabla \cdot(a \nabla u)$. Then it holds that

$$
\max _{1 \leq i \leq N_{s}}\left|\mathcal{L}_{h}^{\text {diff }} u\left(\mathbf{x}_{i}\right)-\mathcal{L}_{l o c}^{\text {diff }} u\left(\mathbf{x}_{i}\right)\right|=O\left(\delta^{2}\right)+O\left(h^{2}\right)
$$

when $\delta>0$ is sufficiently small. 
Proof. Note that

$$
\begin{aligned}
\mathcal{L}_{h}^{\text {diff }} u\left(\mathbf{x}_{i}\right)= & -2 a\left(\mathbf{x}_{i}\right) \int_{B(\mathbf{0}, \delta)} \mathcal{I}_{h}\left(\frac{u\left(\mathbf{x}_{i}+\mathbf{z}\right)-u\left(\mathbf{x}_{i}\right)}{G(\mathbf{z})}\right) G(\mathbf{z}) \gamma_{d}(\|\mathbf{z}\|) d \mathbf{z} \\
& -\int_{B(\mathbf{0}, \delta)} \mathcal{I}_{h}\left(\frac{\left(u\left(\mathbf{x}_{i}+\mathbf{z}\right)-u\left(\mathbf{x}_{i}\right)\right)\left(a\left(\mathbf{x}_{i}+\mathbf{z}\right)-a\left(\mathbf{x}_{i}\right)\right)}{G(\mathbf{z})}\right) G(\mathbf{z}) \gamma_{d}(\|\mathbf{z}\|) d \mathbf{z} .
\end{aligned}
$$

By Taylor expansion, we have

$$
u\left(\mathbf{x}_{i}+\mathbf{z}\right)-u\left(\mathbf{x}_{i}\right)=\nabla u\left(\mathbf{x}_{i}\right)^{T} \mathbf{z}+\frac{1}{2} \mathbf{z}^{T} \boldsymbol{H}_{u}\left(\mathbf{x}_{i}\right) \mathbf{z}+\sum_{p, q, r=1}^{n} \frac{1}{3 !} \frac{\partial^{3} u\left(\mathbf{x}_{i}\right)}{\partial x_{p} \partial x_{q} \partial x_{r}} z_{p} z_{q} z_{r}+R_{1}(\mathbf{z})
$$

and

$$
\begin{aligned}
\left(u\left(\mathbf{x}_{i}+\mathbf{z}\right)-u\left(\mathbf{x}_{i}\right)\right)\left(a(\mathbf{y})-a\left(\mathbf{x}_{i}\right)\right)= & \nabla u\left(\mathbf{x}_{i}\right)^{T} \mathbf{z} \nabla a\left(\mathbf{x}_{i}\right)^{T} \mathbf{z}+\frac{1}{2} \nabla u\left(\mathbf{x}_{i}\right)^{T} \mathbf{z z}^{T} \boldsymbol{H}_{a}\left(\mathbf{x}_{i}\right) \mathbf{z} \\
& +\frac{1}{2} \nabla a\left(\mathbf{x}_{i}\right)^{T} \mathbf{z} \mathbf{z}^{T} \boldsymbol{H}_{u}\left(\mathbf{x}_{i}\right) \mathbf{z}+R_{2}(\mathbf{z})
\end{aligned}
$$

with $R_{1}(\mathbf{z})=O\left(\|\mathbf{z}\|^{4}\right)$ and $R_{2}(\mathbf{z})=O\left(\|\mathbf{z}\|^{4}\right)$, where $\boldsymbol{H}_{u}$ and $\boldsymbol{H}_{a}$ denote the Hessians of the functions $u$ and $a$ respectively.

By the symmetry of the grid around $\mathbf{x}_{i}$, we obtain

$$
-2 a\left(\mathbf{x}_{i}\right) \int_{B(\mathbf{0}, \delta)} \mathcal{I}_{h}\left(\frac{\nabla u\left(\mathbf{x}_{i}^{T}\right) \mathbf{z}}{G(\mathbf{z})}\right) \gamma_{d}(\|\mathbf{z}\|) d \mathbf{z}=0,
$$

and

$$
-2 a\left(\mathbf{x}_{i}\right) \int_{B(\mathbf{0}, \delta)} \mathcal{I}_{h}\left(\frac{\sum_{1 \leq p, q, r \leq 3} \frac{\partial u^{3}\left(\mathbf{x}_{i}\right)}{\partial x_{p} \partial x_{q} \partial x_{r}} z_{p} z_{q} z_{r}}{3 ! G(\mathbf{z})}\right) \gamma_{d}(\|\mathbf{z}\|) d \mathbf{z}=0 .
$$

Let $\mathbf{z}_{j}=\mathbf{x}_{j}-\mathbf{x}_{i}=\left(z_{j 1}, \cdots, z_{j n}\right)$ and $\tilde{\phi}_{j}(\mathbf{z})=\phi_{j}\left(\mathbf{x}_{i}+z\right)$, we have

$$
\begin{aligned}
-2 a\left(\mathbf{x}_{i}\right) \int_{B(\mathbf{0}, \delta)} \mathcal{I}_{h}\left(\frac{\mathbf{z}^{T} \boldsymbol{H}_{u}\left(\mathbf{x}_{i}\right) \mathbf{z}}{2 G(\mathbf{z})}\right) G(\mathbf{z}) \gamma_{d}(\|\mathbf{z}\|) d \mathbf{z} \\
=-2 a\left(\mathbf{x}_{i}\right) \sum_{j \neq i} \frac{\mathbf{z}_{j}^{T} \boldsymbol{H}_{u}\left(\mathbf{x}_{i}\right) \mathbf{z}_{j}}{2 G\left(\mathbf{z}_{j}\right)} \int_{B\left(\mathbf{0}_{i}, \delta\right)} \tilde{\phi}_{j}(\mathbf{z}) G(\mathbf{z}) \gamma_{d}(\|\mathbf{z}\|) d \mathbf{z} \\
=-a\left(\mathbf{x}_{i}\right) \sum_{j \neq i} \frac{\mathbf{z}_{j}^{T} \boldsymbol{H}_{u}\left(\mathbf{x}_{i}\right) \mathbf{z}_{j}}{\left\|\mathbf{z}_{j}\right\|^{2}}\left(\left|z_{j_{1}}\right|+\cdots+\left|z_{j_{n}}\right|\right) Q_{j}
\end{aligned}
$$

where $Q_{j}=\int_{B\left(\mathbf{0}_{i}, \delta\right)} \tilde{\phi}_{j}(\mathbf{z}) G(\mathbf{z}) \gamma_{d}(\|\mathbf{z}\|) d \mathbf{z}$. Due to the symmetry of the grid and $\left\{Q_{j}\right\}$, and the fact that $\sum_{j \neq i}\left(\left|z_{j_{1}}\right|+\cdots+\left|z_{j_{n}}\right|\right) \tilde{\phi}_{j}(\mathbf{z})=\left|z_{1}\right|+\cdots+\left|z_{n}\right|$, we further obtain

$$
-2 a\left(\mathbf{x}_{i}\right) \int_{B(\mathbf{0}, \delta)} \mathcal{I}_{h}\left(\frac{\mathbf{z}^{T} \boldsymbol{H}_{u}\left(\mathbf{x}_{i}\right) \mathbf{z}}{2 G(\mathbf{z})}\right) G(\mathbf{z}) \gamma_{d}(\|\mathbf{z}\|) d \mathbf{z}
$$




$$
\begin{aligned}
& =-a\left(\mathbf{x}_{i}\right) \sum_{j \neq i}\left(\sum_{k=1}^{n} \frac{z_{j_{k}}^{2} \frac{\partial^{2} u}{\partial x_{k}^{2}}\left(\mathbf{x}_{i}\right)}{\left\|\mathbf{z}_{j}\right\|^{2}}\left(\left|z_{j_{1}}\right|+\cdots+\left|z_{j_{n}}\right|\right) Q_{j}\right) \\
& =-a\left(\mathbf{x}_{i}\right) \sum_{k=1}^{n}\left(\sum_{j \neq i} \frac{z_{j_{k}}^{2}}{\left\|\mathbf{z}_{j}\right\|^{2}}\left(\left|z_{j_{1}}\right|+\cdots+\left|z_{j_{n}}\right|\right) Q_{j}\right) \frac{\partial^{2} u}{\partial x_{k}^{2}}\left(\mathbf{x}_{i}\right) \\
& =-\frac{a\left(\mathbf{x}_{i}\right)}{n} \sum_{k=1}^{n}\left(\sum_{j \neq i} \frac{z_{j_{1}}^{2}+\cdots+z_{j_{k}}^{2}}{\left\|\mathbf{z}_{j}\right\|^{2}}\left(\left|z_{j_{1}}\right|+\cdots+\left|z_{j_{n}}\right|\right) Q_{j}\right) \frac{\partial^{2} u}{\partial x_{k}^{2}}\left(\mathbf{x}_{i}\right) \\
& =-\frac{a\left(\mathbf{x}_{i}\right)}{n} \sum_{k=1}^{n}\left(\int_{B(\mathbf{0}, \delta)} \sum_{j \neq i}\left(\left|z_{j_{1}}\right|+\cdots+\left|z_{j_{n}}\right|\right) \tilde{\phi}_{j}(\mathbf{z}) G(\mathbf{z}) \gamma_{d}(\|\mathbf{z}\|) d \mathbf{z}\right) \frac{\partial^{2} u}{\partial x_{k}^{2}}\left(\mathbf{x}_{i}\right) \\
= & -\frac{a\left(\mathbf{x}_{i}\right)}{n} \sum_{k=1}^{n}\left(\int_{B(\mathbf{0}, \delta)}\left(\left|z_{1}\right|+\cdots+\left|z_{n}\right|\right) G(\mathbf{z}) \gamma_{d}(\|\mathbf{z}\|) d \mathbf{z}\right) \frac{\partial^{2} u}{\partial x_{k}^{2}}\left(\mathbf{x}_{i}\right) \\
= & -\frac{a\left(\mathbf{x}_{i}\right)}{n} \sum_{k=1}^{n}\left(\int_{B(\mathbf{0}, \delta)}\|\mathbf{z}\|^{2} \gamma_{d}(\|\mathbf{z}\|) d \mathbf{z}\right) \frac{\partial^{2} u}{\partial x_{k}^{2}}\left(\mathbf{x}_{i}\right) \\
& =-a\left(\mathbf{x}_{i}\right) \sum_{k=1}^{n} \frac{\partial^{2} u}{\partial x_{k}^{2}\left(\mathbf{x}_{i}\right)=-a\left(\mathbf{x}_{i}\right) \Delta u\left(\mathbf{x}_{i}\right) .}
\end{aligned}
$$

By interpolation property, we then have

$$
\begin{aligned}
& -a\left(\mathbf{x}_{i}\right) \int_{B(0, \delta)} \mathcal{I}_{h}\left(\frac{R_{1}(\mathbf{z})}{G(\mathbf{z})}\right) G(\mathbf{z}) \gamma_{d}(\|\mathbf{z}\|) d \mathbf{z} \\
& \quad=-a\left(\mathbf{x}_{i}\right) \int_{B(0, \delta)}\left(R_{1}(\mathbf{z})+O\left(\|\mathbf{z}\|^{2} h^{2}\right)\right) \gamma_{d}(\|\mathbf{z}\|) d \mathbf{z}=O\left(\delta^{2}\right)+O\left(h^{2}\right) .
\end{aligned}
$$

Thus we get by (3.14), (3.15), (3.17) and (3.18) that

$$
\begin{gathered}
-2 a\left(\mathbf{x}_{i}\right) \int_{B(\mathbf{0}, \delta)} \mathcal{I}_{h}\left(\frac{u\left(\mathbf{x}_{i}+\mathbf{z}\right)-u\left(\mathbf{x}_{i}\right)}{G(\mathbf{z})}\right) \gamma_{d}(\|\mathbf{z}\|) d \mathbf{z} \\
=-a\left(\mathbf{x}_{i}\right) \Delta u\left(\mathbf{x}_{i}\right)+O\left(\delta^{2}\right)+O\left(h^{2}\right) .
\end{gathered}
$$

Next we consider the second integral in (3.13). By symmetry of the grid around $\mathbf{x}_{i}$, we first obtain that

$$
\begin{aligned}
& -\int_{B(\mathbf{0}, \delta)} \mathcal{I}_{h}\left(\frac{1}{2} \frac{\nabla u\left(\mathbf{x}_{i}\right)^{T} \mathbf{z z}^{T} \boldsymbol{H}_{a}\left(\mathbf{x}_{i}\right) \mathbf{z}}{G(\mathbf{z})}\right) G(\mathbf{z}) \gamma_{d}(\|\mathbf{z}\|) d \mathbf{z}=0 \\
& -\int_{B(\mathbf{0}, \delta)} \mathcal{I}_{h}\left(\frac{1}{2} \frac{\nabla a\left(\mathbf{x}_{i}\right)^{T} \mathbf{z z}^{T} \boldsymbol{H}_{u}\left(\mathbf{x}_{i}\right) \mathbf{z}}{G(\mathbf{z})}\right) G(\mathbf{z}) \gamma_{d}(\|\mathbf{z}\|) d \mathbf{z}=0 .
\end{aligned}
$$

Furthermore, by similar techniques used in deriving (3.17), we get

$$
-\int_{B(\mathbf{0}, \delta)} \mathcal{I}_{h}\left(\frac{\nabla u\left(\mathbf{x}_{i}\right)^{T} \mathbf{z} \nabla a\left(\mathbf{x}_{i}\right)^{T} \mathbf{z}}{G(\mathbf{z})}\right) G(\mathbf{z}) \gamma_{d}(\|\mathbf{z}\|) d \mathbf{z}
$$




$$
\begin{aligned}
& =-\int_{B(\mathbf{0}, \delta)} \mathcal{I}_{h}\left(\frac{\mathbf{z}^{T}\left(\nabla u\left(\mathbf{x}_{i}\right) \nabla a\left(\mathbf{x}_{i}\right)^{T}\right) \mathbf{z}}{G(\mathbf{z})}\right) G(\mathbf{z}) \gamma_{d}(\|\mathbf{z}\|) d \mathbf{z} \\
& =-\sum_{j \neq i} \frac{\mathbf{z}_{j}^{T}\left(\nabla u \nabla a\left(\mathbf{x}_{i}\right)^{T}\right) \mathbf{z}_{j}}{G\left(\mathbf{z}_{j}\right)} Q_{j} \\
& =-\nabla a\left(\mathbf{x}_{i}\right) \cdot \nabla u\left(\mathbf{x}_{i}\right)
\end{aligned}
$$

and

$$
\begin{aligned}
-\int_{B(\mathbf{0}, \delta)} & \mathcal{I}_{h}\left(\frac{R_{2}(\mathbf{z})}{G(\mathbf{z})}\right) G(\mathbf{z}) \gamma_{d}(\|\mathbf{z}\|) d \mathbf{z} \\
\quad= & \int_{B(\mathbf{0}, \delta)}\left(R_{2}(\mathbf{z})+O\left(\|\mathbf{z}\|^{2} h^{2}\right)\right) \gamma_{d}(\|\mathbf{z}\|) d \mathbf{z}=O\left(\delta^{2}\right)+O\left(h^{2}\right) .
\end{aligned}
$$

Thus by (3.20), (3.21), (3.22) and (3.23) we obtain that

$$
\begin{gathered}
-\int_{B(\mathbf{0}, \delta)} \mathcal{I}_{h}\left(\frac{\left(u\left(\mathbf{x}_{i}+\mathbf{z}\right)-u\left(\mathbf{x}_{i}\right)\right)\left(a\left(\mathbf{x}_{i}+\mathbf{z}\right)-a\left(\mathbf{x}_{i}\right)\right)}{G(\mathbf{z})}\right) G(\mathbf{z}) \gamma_{d}(\|\mathbf{z}\|) d \mathbf{z} \\
=-\nabla a\left(\mathbf{x}_{i}\right) \cdot \nabla u\left(\mathbf{x}_{i}\right)+O\left(\delta^{2}\right)+O\left(h^{2}\right) .
\end{gathered}
$$

By combining (3.13), (3.19) and (3.24), we obtain

$$
\mathcal{L}_{h}^{\text {diff }} u\left(\mathbf{x}_{i}\right)=-\nabla\left(a\left(\mathbf{x}_{i}\right) \nabla u\left(\mathbf{x}_{i}\right)\right)+O\left(\delta^{2}\right)+O\left(h^{2}\right),
$$

which gives us (3.12).

Now we turn to the approximate convection at each node $\mathbf{x}_{i}$ defined by (3.5). In order to simplify the analysis, we remove the singularity of the velocity function by further assuming in the remaining part of the paper that $\|\boldsymbol{b}\| \geq b_{0}>0$ in $\bar{\Omega}$ for some constant $b_{0}$ when there is a non-constant convection velocity and $\boldsymbol{b} \in\left(C^{1}(\bar{\Omega})\right)^{n}$. For convenience of notation, we also define $H B_{\mathbf{x}}(\mathbf{0}, \delta)=$ $\{\mathbf{z} \mid \mathbf{x}+\mathbf{z} \in H B(\mathbf{x}, \delta)\}, \widetilde{H B}_{\mathbf{x}}(\mathbf{0}, \delta)=\{\mathbf{z} \mid \mathbf{x}+\mathbf{z} \in \widetilde{H B}(\mathbf{x}, \delta)\}$ and $\widehat{H B}_{\mathbf{x}}(\mathbf{0}, \delta)=\{\mathbf{z} \mid \mathbf{x}+\mathbf{z} \in \widehat{H B}(\mathbf{x}, \delta)\}$. Under such a regularity assumption on $\boldsymbol{b}$, there exist constants $c_{1}$ and $c_{2}=c_{1} / b_{0}$ such that

$$
\|\boldsymbol{b}(\mathbf{y})-\boldsymbol{b}(\mathbf{x})\| \leq c_{1}\|\mathbf{x}-\mathbf{y}\| \leq c_{2} \min (\|\boldsymbol{b}(\mathbf{x})\|,\|\boldsymbol{b}(\mathbf{y})\|)\|\mathbf{x}-\mathbf{y}\|
$$

for any $\mathbf{x}, \mathbf{y} \in \bar{\Omega}$. We choose $\delta$ small enough so that $c_{2} \delta<1 / 2$. For any two points $\mathbf{x}, \mathbf{y} \in \bar{\Omega}$ with $\|\mathbf{x}-\mathbf{y}\| \leq \delta$, let $\psi$ denote the angle between the vectors $\boldsymbol{b}(\mathbf{x})$ and $\boldsymbol{b}(\mathbf{y})$, then clearly $\psi$ must be acute since $\|\boldsymbol{b}(\mathbf{y})-\boldsymbol{b}(\mathbf{x})\| \leq \min (\|\boldsymbol{b}(\mathbf{x})\|,\|\boldsymbol{b}(\mathbf{y})\|) / 2$. We also have

$$
\sin \psi \leq \frac{c_{2}\|\boldsymbol{b}(\mathbf{x})\|\|\mathbf{x}-\mathbf{y}\|}{\|\boldsymbol{b}(\mathbf{x})\|} \leq c_{2}\|\mathbf{x}-\mathbf{y}\|<1 / 2
$$

and consequently $\tan (\psi) \leq 2 c_{2}\|\mathbf{x}-\mathbf{y}\|$. This concludes that, for any $0<r \leq \delta$,

$$
\begin{aligned}
\int_{\partial B(\mathbf{x}, r)} & \left|1_{H B(\mathbf{x}, \delta)}(\mathbf{y})-1_{\widehat{H B}(\mathbf{x}, \delta)}(\mathbf{y})\right| d S(\mathbf{y}) \\
\leq & r \tan (\psi)\left(\beta_{n-1} r^{n-2}\right) \leq r\left(2 c_{2} r\right) \beta_{n-1} r^{n-1} \leq c_{3} r^{n}
\end{aligned}
$$


where $c_{3}=2 c_{2} \beta_{n-1}$. In addition, for any $\mathbf{y} \in(H B(\mathbf{x}, \delta)-\widehat{H B}(\mathbf{x}, \delta)) \cup(\widehat{H B}(\mathbf{x}, \delta)-H B(\mathbf{x}, \delta))$, we have

$$
\begin{aligned}
\frac{\left|\boldsymbol{b}(\mathbf{y})^{T}(\mathbf{y}-\mathbf{x})\right|}{\|\mathbf{y}-\mathbf{x}\|} & \leq\|\boldsymbol{b}(\mathbf{y})\| \cos (\pi / 2-\psi) \\
& =\|\boldsymbol{b}(\mathbf{y})\| \sin (\psi) \mid \leq c_{2}\|\boldsymbol{b}(\mathbf{y})\|\|\mathbf{x}-\mathbf{y}\| \leq c_{4} \delta
\end{aligned}
$$

where $c_{4}=c_{2} \max _{\mathbf{y} \in \Omega}\|\boldsymbol{b}(\mathbf{y})\|$.

Lemma 1. Assume that $\boldsymbol{b} \in\left(C^{2}(\bar{\Omega})\right)^{n}$. It holds that

$$
\max _{1 \leq i \leq N_{s}}\left|\mathcal{D}(\boldsymbol{\mu})\left(\mathbf{x}_{i}\right)-\nabla \cdot \boldsymbol{b}\left(\mathbf{x}_{i}\right)\right|=O(\delta)
$$

when $\delta>0$ is sufficiently small.

Proof. Note that

$$
\begin{aligned}
\mathcal{D}(\boldsymbol{\mu})\left(\mathbf{x}_{i}\right)= & \int_{\Omega}\left(\boldsymbol{b}(\mathbf{y}) \omega_{c}\left(\mathbf{y}, \mathbf{x}_{i}\right)+\boldsymbol{b}\left(\mathbf{x}_{i}\right) \omega_{c}\left(\mathbf{x}_{i}, \mathbf{y}\right)\right)^{T} \frac{\mathbf{y}-\mathbf{x}_{i}}{\left\|\mathbf{y}-\mathbf{x}_{i}\right\|} d \mathbf{y} \\
= & \int_{B\left(\mathbf{x}_{i}, \delta\right)}\left(\boldsymbol{b}(\mathbf{y}) 1_{H B\left(\mathbf{x}_{i}, \delta\right)}(\mathbf{y})-\boldsymbol{b}\left(\mathbf{x}_{i}\right) 1_{\widehat{H B}\left(\mathbf{x}_{i}, \delta\right)}(\mathbf{y})\right)^{T} \frac{\mathbf{y}-\mathbf{x}_{i}}{\left\|\mathbf{y}-\mathbf{x}_{i}\right\|} \gamma_{c}\left(\left\|\mathbf{y}-\mathbf{x}_{i}\right\|\right) d \mathbf{y} \\
= & \int_{\widehat{H B}\left(\mathbf{x}_{i}, \delta\right)}\left(\boldsymbol{b}(\mathbf{y})-\boldsymbol{b}\left(\mathbf{x}_{i}\right)\right)^{T} \frac{\mathbf{y}-\mathbf{x}_{i}}{\left\|\mathbf{y}-\mathbf{x}_{i}\right\|} \gamma_{c}\left(\left\|\mathbf{y}-\mathbf{x}_{i}\right\|\right) d \mathbf{y} \\
& +\int_{B\left(\mathbf{x}_{i}, \delta\right)} \boldsymbol{b}(\mathbf{y})^{T} \frac{\mathbf{y}-\mathbf{x}_{i}}{\left\|\mathbf{y}-\mathbf{x}_{i}\right\|}\left(1_{H B\left(\mathbf{x}_{i}, \delta\right)}(\mathbf{y})-1_{\widehat{H B}\left(\mathbf{x}_{i}, \delta\right)}(\mathbf{y})\right) \gamma_{c}\left(\left\|\mathbf{y}-\mathbf{x}_{i}\right\|\right) d \mathbf{y} .
\end{aligned}
$$

It is easy to find that

$$
\begin{aligned}
\int_{\widehat{H B}\left(\mathbf{x}_{i}, \delta\right)} & \left(\boldsymbol{b}(\mathbf{y})-\boldsymbol{b}\left(\mathbf{x}_{i}\right)\right)^{T} \frac{\mathbf{y}-\mathbf{x}_{i}}{\left\|\mathbf{y}-\mathbf{x}_{i}\right\|} \gamma_{c}\left(\left\|\mathbf{y}-\mathbf{x}_{i}\right\|\right) d \mathbf{y} \\
= & \int_{\widehat{H B}\left(\mathbf{x}_{i}, \delta\right)}\left(\mathbf{z}^{T} \nabla \boldsymbol{b}\left(\mathbf{x}_{i}\right) \mathbf{z}+O(\|\mathbf{z}\|)^{3}\right) \frac{\gamma_{c}(\|\mathbf{z}\|)}{\|\mathbf{z}\|} d \mathbf{z} \\
= & \nabla \cdot \boldsymbol{b}\left(\mathbf{x}_{i}\right)+O(\delta) .
\end{aligned}
$$

By using (3.26) and (3.27), we have

$$
\begin{aligned}
& \left|\int_{B\left(\mathbf{x}_{i}, \delta\right)} \boldsymbol{b}(\mathbf{y})^{T} \frac{\mathbf{y}-\mathbf{x}_{i}}{\left\|\mathbf{y}-\mathbf{x}_{i}\right\|}\left(1_{H B\left(\mathbf{x}_{i}, \delta\right)}(\mathbf{y})-1_{\widehat{H B}\left(\mathbf{x}_{i}, \delta\right)}(\mathbf{y})\right) \gamma_{c}\left(\left\|\mathbf{y}-\mathbf{x}_{i}\right\|\right) d \mathbf{y}\right| \\
& \quad \leq c_{4} \delta \int_{B\left(\mathbf{x}_{i}, \delta\right)}\left|1_{H B\left(\mathbf{x}_{i}, \delta\right)}(\mathbf{y})-1_{\widehat{H B}\left(\mathbf{x}_{i}, \delta\right)}(\mathbf{y})\right| \gamma_{c}\left(\left\|\mathbf{y}-\mathbf{x}_{i}\right\|\right) d \mathbf{y} \\
& \quad=c_{4} \delta \int_{0}^{\delta} \int_{\partial B\left(\mathbf{x}_{i}, r\right)}\left|1_{H B\left(\mathbf{x}_{i}, \delta\right)}(\mathbf{y})-1_{\widehat{H B}\left(\mathbf{x}_{i}, \delta\right)}(\mathbf{y})\right| \gamma_{c}(r) d S(\mathbf{y}) d r \\
& \quad \leq c_{3} c_{4} \delta \int_{0}^{\delta} r^{n} \gamma_{c}(r) d r \\
& \leq c_{5} \delta=O(\delta) .
\end{aligned}
$$

Thus we obtain (3.28) from (3.29),(3.30) and (3.31). 
Theorem 7. Assume that $u \in C^{3}(\bar{\Omega}), \boldsymbol{b} \in\left(C^{2}(\bar{\Omega})^{n}\right.$ and let $\mathcal{L}_{h}^{\text {conv }} u\left(\mathbf{x}_{i}\right)=\nabla \cdot\left(\boldsymbol{b}\left(\mathbf{x}_{i}\right) u\left(\mathbf{x}_{i}\right)\right)$. Then it holds that

$$
\max _{1 \leq i \leq N_{s}}\left|\mathcal{L}_{h}^{\text {conv }} u\left(\mathbf{x}_{i}\right)-\mathcal{L}_{\text {loc }}^{\text {conv }} u\left(\mathbf{x}_{i}\right)\right|=O(\delta)+O\left(h^{2}\right)
$$

when $\delta>0$ and $h>0$ are sufficiently small.

Proof. Note that

$$
\begin{aligned}
& \mathcal{L}_{h}^{\text {conv }} u\left(\mathbf{x}_{i}\right)=\int_{H B_{\mathbf{x}_{i}}(\mathbf{0}, \delta)} \mathcal{I}_{h}\left(u\left(\mathbf{x}_{i}+\mathbf{z}\right)-u\left(\mathbf{x}_{i}\right)\right) \boldsymbol{b}\left(\mathbf{x}_{i}+\mathbf{z}\right)^{T} \frac{\mathbf{z}}{\|\mathbf{z}\|} \gamma_{c}(\|\mathbf{z}\|) d \mathbf{y} \\
& +u\left(\mathbf{x}_{i}\right) \mathcal{D}(\boldsymbol{\mu})\left(\mathbf{x}_{i}\right) \\
& =\int_{\widehat{H B}} \mathcal{I}_{h}\left(u\left(\mathbf{x}_{i}+\mathbf{z}\right)-u\left(\mathbf{x}_{i}\right)\right) \boldsymbol{b}\left(\mathbf{x}_{i}+\mathbf{z}\right)^{T} \frac{\mathbf{z}}{\|\mathbf{z}\|} \gamma_{c}(\|\mathbf{z}\|) d \mathbf{z} \\
& +\int_{B\left(\mathbf{x}_{i}, \delta\right)} \mathcal{I}_{h}\left(u(\mathbf{y})-u\left(\mathbf{x}_{i}\right)\right)\left(1_{H B\left(\mathbf{x}_{i}, \delta\right)}(\mathbf{y})-1_{\widehat{H B}\left(\mathbf{x}_{i}, \delta\right)}(\mathbf{y})\right) \\
& \boldsymbol{b}(\mathbf{y})^{T} \frac{\mathbf{y}-\mathbf{x}}{\|\mathbf{y}-\mathbf{x}\|} \gamma_{c}(\|\mathbf{z}\|) d \mathbf{y} \\
& +u\left(\mathbf{x}_{i}\right) \mathcal{D}(\boldsymbol{\mu})\left(\mathbf{x}_{i}\right) .
\end{aligned}
$$

By Taylor expansion, we have

$$
\begin{aligned}
u\left(\mathbf{x}_{i}+\mathbf{z}\right)-u\left(\mathbf{x}_{i}\right) & =\nabla u\left(\mathbf{x}_{i}\right)^{T} \mathbf{z}+\mathbf{z}^{T} \boldsymbol{H}_{u}\left(\mathbf{x}_{i}\right)^{T} \mathbf{z}+R_{3}(\mathbf{z}) \\
& =\nabla u\left(\mathbf{x}_{i}\right)^{T} \mathbf{z}+R_{4}(\mathbf{z})
\end{aligned}
$$

where $R_{3}(\mathbf{z})=O\left(\|\mathbf{z}\|^{3}\right)$ and $R_{4}(\mathbf{z})=O\left(\|\mathbf{z}\|^{2}\right)$. Using the linearity of $\nabla u\left(\mathbf{x}_{i}\right)^{T} \mathbf{z}$, it can be shown that

$$
\begin{aligned}
\int_{\widehat{H B}_{\mathbf{x}_{i}}(\mathbf{0}, \delta)} & \mathcal{I}_{h}\left(\nabla u\left(\mathbf{x}_{i}\right)^{T} \mathbf{z}\right) \boldsymbol{b}\left(\mathbf{x}_{i}+\mathbf{z}\right)^{T} \frac{\mathbf{z}}{\|\mathbf{z}\|} \gamma_{c}(\|\mathbf{z}\|) d \mathbf{z} \\
= & \int_{\widehat{H B}_{\mathbf{x}_{i}}(\mathbf{0}, \delta)} \nabla u\left(\mathbf{x}_{i}\right)^{T} \mathbf{z z}^{T}\left(\boldsymbol{b}\left(\mathbf{x}_{i}\right)+O(\|\mathbf{z}\|)\right) \frac{1}{\|\mathbf{z}\|} \gamma_{c}(\|\mathbf{z}\|) d \mathbf{z} \\
= & \boldsymbol{b}\left(\mathbf{x}_{i}\right) \cdot \nabla u\left(\mathbf{x}_{i}\right)+O(\delta),
\end{aligned}
$$

and it is also easy to find that

$$
\begin{array}{r}
\int_{\widehat{H B}_{\mathbf{x}_{i}}(\mathbf{0}, \delta)} \mathcal{I}_{h}\left(\mathbf{z}^{T} \boldsymbol{H}_{u}\left(\mathbf{x}_{i}\right)^{T} \mathbf{z}\right) \boldsymbol{b}\left(\mathbf{x}_{i}+\mathbf{z}\right)^{T} \frac{\mathbf{z}}{\|\mathbf{z}\|} \gamma_{c}(\|\mathbf{z}\|) d \mathbf{z} \\
=\int_{\widehat{H B}_{\mathbf{x}_{i}}(\mathbf{0}, \delta)}\left(O\left(\|\mathbf{z}\|^{2}\right)\right) \frac{\mathbf{z}}{\|\mathbf{z}\|} \gamma_{c}(\|\mathbf{z}\|) d \mathbf{z}=O(\delta)
\end{array}
$$

and

$$
\begin{aligned}
\int_{\widehat{H B}_{\mathbf{x}_{i}}(\mathbf{0}, \delta)} & \mathcal{I}_{h}\left(R_{3}(\mathbf{z})\right) \boldsymbol{b}\left(\mathbf{x}_{i}+\mathbf{z}\right)^{T} \frac{\mathbf{z}}{\|\mathbf{z}\|} \gamma_{c}(\|\mathbf{z}\|) d \mathbf{z} \\
= & \int_{\widehat{H B}_{\mathbf{x}_{i}}(\mathbf{0}, \delta)}\left(R_{3}(\mathbf{z})+O\left(\|\mathbf{z}\| h^{2}\right)\right)\left(\boldsymbol{b}\left(\mathbf{x}_{i}\right)+O(\|\mathbf{z}\|)^{T} \frac{\mathbf{z}}{\|\mathbf{z}\|} \gamma_{c}(\|\mathbf{z}\|) d \mathbf{z}\right.
\end{aligned}
$$




$$
=O\left(\delta^{2}\right)+O\left(h^{2}\right)
$$

Thus we have from (3.34), (3.35) and (3.36) that

$$
\begin{gathered}
\int_{\widehat{H B}_{\mathbf{x}_{i}}(\mathbf{0}, \delta)} \mathcal{I}_{h}\left(u\left(\mathbf{x}_{i}+\mathbf{z}\right)-u\left(\mathbf{x}_{i}\right)\right) \boldsymbol{b}\left(\mathbf{x}_{i}+\mathbf{z}\right)^{T} \frac{\mathbf{z}}{\|\mathbf{z}\|} \gamma_{c}(\|\mathbf{z}\|) d \mathbf{z} \\
=\boldsymbol{b}\left(\mathbf{x}_{i}\right) \cdot \nabla u\left(\mathbf{x}_{i}\right)+O(\delta)+O\left(h^{2}\right) .
\end{gathered}
$$

By analysis similar to that in Lemma 1, it holds that

$$
\begin{aligned}
& \int_{B\left(\mathbf{x}_{i}, \delta\right)} \mathcal{I}_{h}\left(\nabla u\left(\mathbf{x}_{i}\right)^{T} \mathbf{z}\right)\left(1_{H B\left(\mathbf{x}_{i}, \delta\right)}(\mathbf{y})-1_{\widehat{H B}\left(\mathbf{x}_{i}, \delta\right)}(\mathbf{y})\right) \frac{\boldsymbol{b}(\mathbf{y})^{T}(\mathbf{y}-\mathbf{x})}{\|\mathbf{y}-\mathbf{x}\|} \gamma_{c}(\|\mathbf{y}-\mathbf{x}\|) d \mathbf{z} \\
& \quad=\int_{B(\mathbf{y}, \delta)}\left(1_{H B\left(\mathbf{x}_{i}, \delta\right)}(\mathbf{y})-1_{\widehat{H B}\left(\mathbf{x}_{i}, \delta\right)}(\mathbf{y})\right) \frac{\nabla u\left(\mathbf{x}_{i}\right)^{T}(\mathbf{y}-\mathbf{x})(\mathbf{y}-\mathbf{x})^{T} \boldsymbol{b}(\mathbf{y})}{\|\mathbf{y}-\mathbf{x}\|} \gamma_{c}(\|\mathbf{y}-\mathbf{x}\|) d \mathbf{y} \\
& \quad=O(\delta)
\end{aligned}
$$

and

$$
\begin{aligned}
\int_{B\left(\mathbf{x}_{i}, \delta\right)} & \mathcal{I}_{h}\left(R_{4}(\mathbf{z})\right)\left(1_{H B\left(\mathbf{x}_{i}, \delta\right)}(\mathbf{y})-1_{\widehat{H B}\left(\mathbf{x}_{i}, \delta\right)}(\mathbf{y})\right) \frac{\boldsymbol{b}(\mathbf{y})^{T}(\mathbf{y}-\mathbf{x})}{\|\mathbf{y}-\mathbf{x}\|} \gamma_{c}(\|\mathbf{y}-\mathbf{x}\|) d \mathbf{z} \\
= & \int_{B(\mathbf{y}, \delta)}\left(R_{4}(\mathbf{z})+O\left(h^{2}\right)\right)\left(1_{H B\left(\mathbf{x}_{i}, \delta\right)}(\mathbf{y})-1_{\widehat{H B}\left(\mathbf{x}_{i}, \delta\right)}(\mathbf{y})\right) \\
& \frac{\nabla u\left(\mathbf{x}_{i}\right)^{T}(\mathbf{y}-\mathbf{x})(\mathbf{y}-\mathbf{x})^{T} \mathbf{b}(\mathbf{y})}{\|\mathbf{y}-\mathbf{x}\|} \gamma_{c}(\|\mathbf{y}-\mathbf{x}\|) d \mathbf{y} \\
= & O\left(\delta^{2}\right)+O\left(h^{2}\right) .
\end{aligned}
$$

Thus we get from (3.38) and (3.39) that

$$
\begin{aligned}
& \int_{B\left(\mathbf{x}_{i}, \delta\right)} \mathcal{I}_{h}\left(u(\mathbf{y})-u\left(\mathbf{x}_{i}\right)\right)\left(1_{H B\left(\mathbf{x}_{i}, \delta\right)}(\mathbf{y})-1_{\widehat{H B}\left(\mathbf{x}_{i}, \delta\right)}(\mathbf{y})\right) \boldsymbol{b}(\mathbf{y})^{T} \frac{\mathbf{y}-\mathbf{x}}{\|\mathbf{y}-\mathbf{x}\|} \gamma_{c}(\|\mathbf{z}\|) d \mathbf{y} \\
& =O(\delta)+O\left(h^{2}\right)
\end{aligned}
$$

The combination of (3.37), (3.40) and Lemma 1 finally gives us (3.32).

Remark 4. We summarize below some observations on the convergence and asymptotic compatibility of the proposed quadrature-based finite difference discretization (3.7) based on Theorems 6 and 7 :

- The quadrature-based finite difference discretization is asymptotically compatible [42], i.e., the approximate solutions always converge to the exact solution of the corresponding local problem when $\delta \rightarrow 0$ and $h \rightarrow 0$ simultaneously in arbitrary manner.

- For the pure nonlocal diffusion problem, the optimal $\delta$-convergence (with $\delta / h=O(1)$ and with respect to the grid refinement) would be of second order in $h$; when both the nonlocal diffusion and convection terms exist in the problem, the $\delta$-convergence is at best of first order, especially in the convection-dominated case. 


\section{Numerical experiments}

In this section, we perform some numerical experiments in the two dimensional space $(n=2)$ to illustrate the conservative nonlocal convection-diffusion model and verify the effectiveness of the quadrature-based finite difference discretization (3.7). The domain $\Omega_{s}=(0,1) \times(0,1)$ is used for all examples. The parameters $s_{d}, s_{c}$ in the nonlocal kernel functions are taken to be $s_{d}=s_{c}=1$, which is a common choice in many existing nonlocal convection and diffusion models. For simplicity, the diffusion coefficient is taken to be a constant as $a\left(x_{1}, x_{2}\right) \equiv a$ with $a=1$ and $a=10^{-7}$ respectively, In comparison, the latter one represents a highly convection-dominated nonlocal problem.

\subsection{Tests with fixed horizon}

We first regard the horizon $\delta$ as a material parameter so that its value is independent of the grid size. We choose the fixed horizon $\delta=0.2$ for all experiments in this subsection. While keeping $\delta$ fixed, the numerical solutions are expected to converge to the exact solution of the nonlocal problem with at best second order as $h \rightarrow 0$.

Example 1. In this example we take a smooth exact solution $u\left(x_{1}, x_{2}\right)=x_{1}^{3}+x_{2}^{2}$. We test two different velocity functions: one is a constant vector $\boldsymbol{b}\left(x_{1}, x_{2}\right)=(\cos (\pi / 4) \text {, } \sin (\pi / 4))^{T}$, the other is $\boldsymbol{b}\left(x_{1}, x_{2}\right)=\left(-x_{1}+5, x_{2}+5\right)^{T}$ that has a small but smooth variation with respect to the coordinates. The right hand side of the nonlocal equation (2.13), $f\left(x_{1}, x_{2}\right)$, is then calculated according to the terms on the left hand side. The boundary condition $g\left(x_{1}, x_{2}\right)$ is imposed by the given exact solution $u\left(x_{1}, x_{2}\right)$.

A uniform rectangular grid of size $N \times N$ is used for the domain $[0,1] \times[0,1]$. We take $N=$ $10,20,40,80$ respectively and solve the problem (2.13) using the proposed quadrature-based finite difference discretization scheme (3.7) on the uniform grids. Numerical results on the solution errors measured by the $L^{\infty}$ and $L^{2}$ norms are reported in Table 4.1. For the constant velocity cases, the $L^{2}$ errors show approximately second order convergence as predicted by the theoretical analysis. For exceedingly small $\delta$, the convergence orders are slightly below the optimal quadratic rates, which might be caused by the inexact calculations of the integrals involving the singular influence functions in our numerical implementation.

Example 2. In this example, a constant velocity $\boldsymbol{b}\left(x_{1}, x_{2}\right)=(\cos (\theta), \sin (\theta))^{T}$ is used, where $\theta$ is a constant angle that determines the constant wind direction. We impose a Dirichlet volumeconstraint condition (as shown in Figure 2): $u\left(x_{1}, x_{2}\right)=1$ in the blue region and $u(x, y)=0$ in the white region. The right hand side function is chosen as $f\left(x_{1}, x_{2}\right)=0$. Although the exact solution is unknown, it clearly takes values between 0 and 1 and there exists a boundary layer ( $a$ rapid change from 1 to 0$)$ in the solution when the diffusion coefficient a is small.

We solve the nonlocal model on a $40 \times 40$ grid using the finite difference discretization, and Fig. 3 shows the simulation results. The top row of Fig. 3 gives the approximate solutions for the case of $a=1$ with $\theta=\pi / 4$ (left) and $\theta=5 \pi / 4$ (right) respectively. It is easy to see that there is no numerical oscillations at all and the maximum principle is well-satisfied by the approximate solution with values varying from 0 to 1 . The bottom row of Fig. 3 presents the approximate solutions for the strongly convection-dominated case of $a=10^{-7}$. We can see that the maximum principle is again well preserved in both numerical simulations. The discrete solutions have a boundary layer close to $x=0$ and $y=0$ as expected in the case $\theta=\pi / 4$ and a boundary layer close to $x=1$ and $y=1$ in the case $\theta=5 \pi / 4$. 


\begin{tabular}{|c|c|c|c|c|c|c|c|c|}
\hline & \multicolumn{8}{|c|}{$\boldsymbol{b}\left(x_{1}, x_{2}\right)=(\cos (\pi / 4), \sin (\pi / 4))^{T}$} \\
\hline & \multicolumn{4}{|c|}{$a=1$} & \multicolumn{4}{|c|}{$a=10^{-7}$} \\
\hline$h$ & $\left\|e^{h}\right\|_{\infty}$ & CR & $\left\|e^{h}\right\|_{2}$ & CR & $\left\|e^{h}\right\|_{\infty}$ & CR & $\left\|e^{h}\right\|_{2}$ & CR \\
\hline $1 / 10$ & $7.7398 \mathrm{e}-02$ & - & $1.3354 \mathrm{e}-02$ & - & $4.7625 \mathrm{e}-02$ & - & $1.0844 \mathrm{e}-02$ & - \\
\hline $1 / 20$ & $2.2019 \mathrm{e}-02$ & 1.81 & $3.2393 \mathrm{e}-03$ & 2.04 & $1.1872 \mathrm{e}-02$ & 2.00 & $2.7798 \mathrm{e}-03$ & 1.96 \\
\hline $1 / 40$ & $6.4225 \mathrm{e}-03$ & 1.78 & $9.0043 \mathrm{e}-04$ & 1.85 & $3.5629 \mathrm{e}-03$ & 1.74 & $7.1472 \mathrm{e}-04$ & 1.95 \\
\hline \multirow[t]{3}{*}{$1 / 80$} & $2.0885 \mathrm{e}-03$ & 1.62 & $2.6126 \mathrm{e}-04$ & 1.78 & $1.2115 \mathrm{e}-03$ & 1.56 & $1.9124 \mathrm{e}-04$ & 1.90 \\
\hline & \multicolumn{8}{|c|}{$\bar{b}\left(x_{1}, x_{2}\right)=\left(-x_{1}+5, x_{2}+5\right)^{T}$} \\
\hline & \multicolumn{4}{|c|}{$a=1$} & \multicolumn{4}{|c|}{$a=10^{-7}$} \\
\hline$h$ & $\left\|e^{h}\right\|_{\infty}$ & $\mathrm{CR}$ & $\left\|e^{h}\right\|_{2}$ & CR & $\left\|e^{h}\right\|_{\infty}$ & CR & $\left\|e^{h}\right\|_{2}$ & $\mathrm{CR}$ \\
\hline $1 / 10$ & $4.0751 \mathrm{e}-02$ & - & $2.3354 \mathrm{e}-02$ & - & $3.9382 \mathrm{e}-02$ & - & $2.6966 \mathrm{e}-02$ & - \\
\hline $1 / 20$ & $1.2082 \mathrm{e}-02$ & 1.71 & $6.5637 \mathrm{e}-03$ & 1.83 & $1.1612 \mathrm{e}-02$ & 1.76 & $7.5512 \mathrm{e}-03$ & 1.84 \\
\hline $1 / 40$ & $3.4391 \mathrm{e}-03$ & 1.81 & $2.1015 \mathrm{e}-03$ & 1.64 & $3.1687 \mathrm{e}-03$ & 1.87 & $2.2198 \mathrm{e}-03$ & 1.76 \\
\hline $1 / 80$ & $9.9902 \mathrm{e}-04$ & 1.78 & $6.9245 \mathrm{e}-04$ & 1.60 & $8.7071 \mathrm{e}-04$ & 1.86 & $7.0298 \mathrm{e}-04$ & 1.66 \\
\hline
\end{tabular}

Table 1: Numerical results on the solution errors and convergence rates by the quadrature-based finite difference discretization in Example 1.

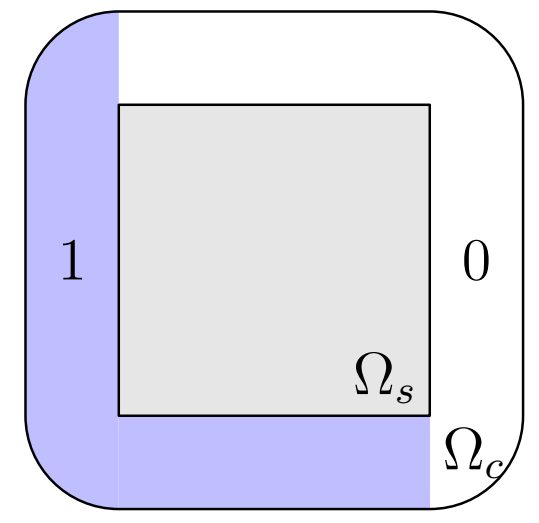

Figure 2: Illustration of the volume-constraint condition for Example 2.

Example 3. The settings for this example are essentially the same as those for Example 2 except that a variable velocity field is taken to be piecewise constant with the discontinuities along the line $x_{1}+x_{2}=1$ (as shown in Fig. 4). In other words, we have $\theta=\theta_{1}$ In the blue region and $\theta=\theta_{2}$ in the white region where $\theta_{1} \neq \theta_{2}$.

We here only test the strongly convection-dominated case with $a=10^{-7}$. Four sets of values for the angles $\left(\theta_{1}, \theta_{2}\right)$ are selected: $(\pi / 4,5 \pi / 4),(5 \pi / 4, \pi / 4),(7 \pi / 4,3 \pi / 4)$, and $(\pi / 4,3 \pi / 4)$. Then we solve the nonlocal problems using the difference approximations on a $40 \times 40$ grid. The simulation results are shown in Fig. 5. We again observe that even with discontinuous velocity fields, our difference scheme is very stable and the maximum principle is well-satisfied by all approximate solutions for the nonlocal convection-diffusion problem.

\subsection{Tests for $\delta$-convergence}

We now investigate the $\delta$-convergence of the proposed quadrature-based finite difference discretization, As the horizon $\delta$ and the grid size $h$ go to zero at a fixed ratio, the approximate solutions of the nonlocal problem (2.13) are expected to converge to that of the corresponding classical local problem (2.15). 

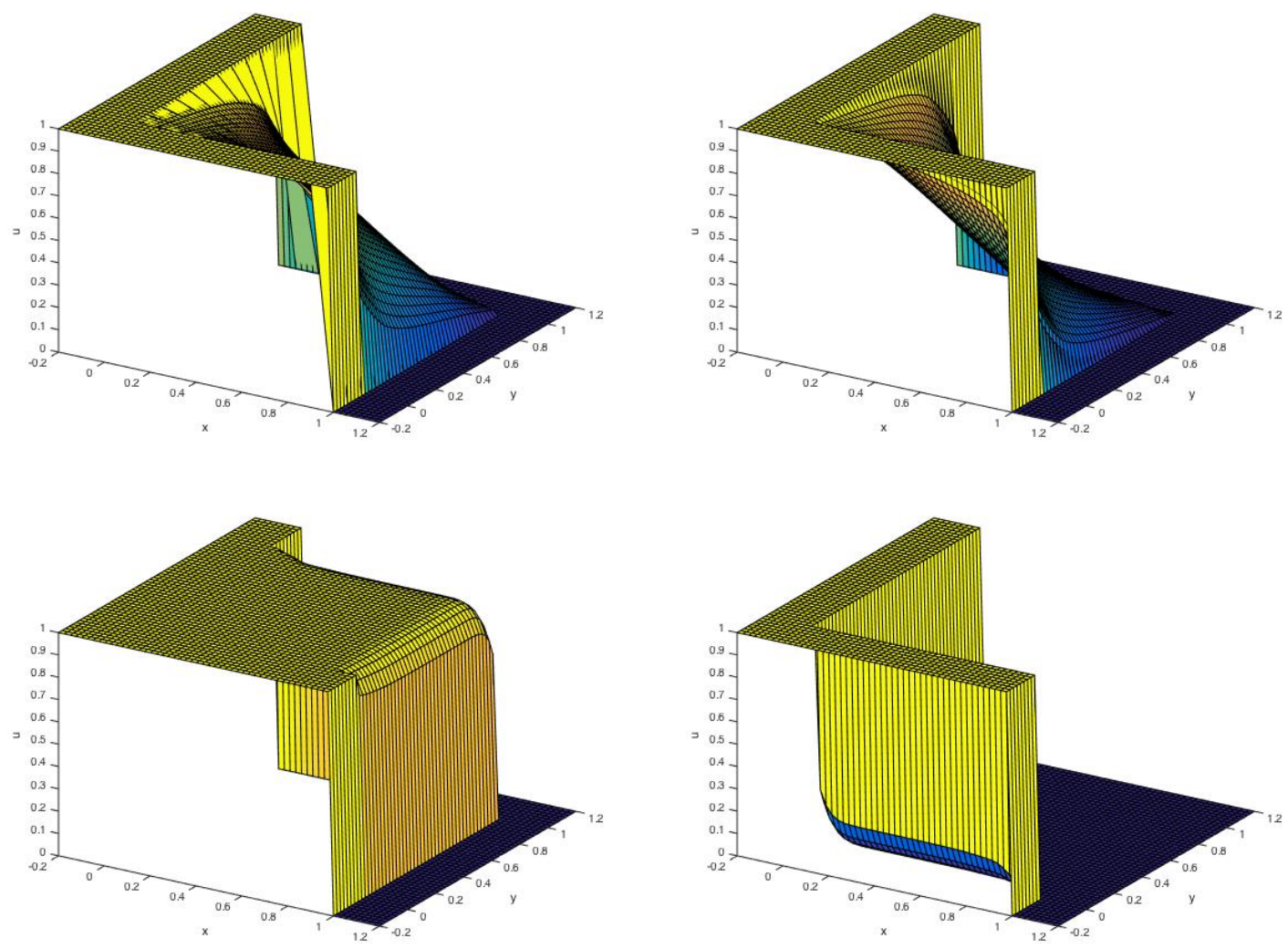

Figure 3: Approximate solutions of the nonlocal convection-diffusion problem with different wind direction $\theta=\pi / 4$ (left column) and $\theta=5 \pi / 4$ (right column) by the difference discretization in Example 2. Top row: $a=1$; bottom row: $a=10^{-7}$.

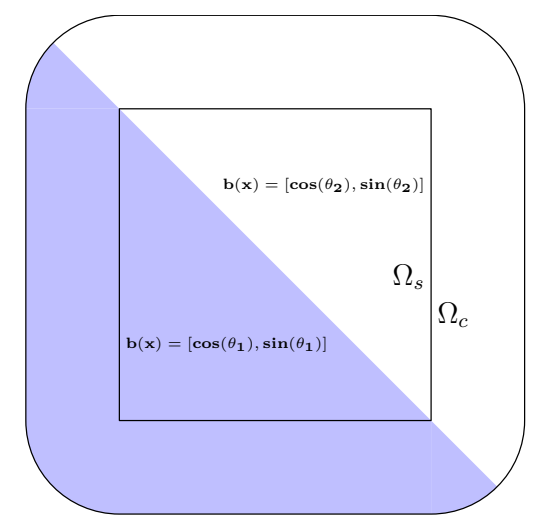

Figure 4: Illustration of the discontinuous velocity field for Example 3.

Example 4. In the example, we take the exact solution $u\left(x_{1}, x_{2}\right)=x_{1}^{3} x_{2}^{3}$ and set the velocity field $\boldsymbol{b}\left(x_{1}, x_{2}\right)=(\cos (\pi / 4), \sin (\pi / 4))^{T}$. The right hand side function $f$ is again given based on the corresponding local limit problem.

We set a fixed ratio $\frac{\delta}{h}=5$ in this example by halving the values of $\delta$ and $h$ simultaneously 

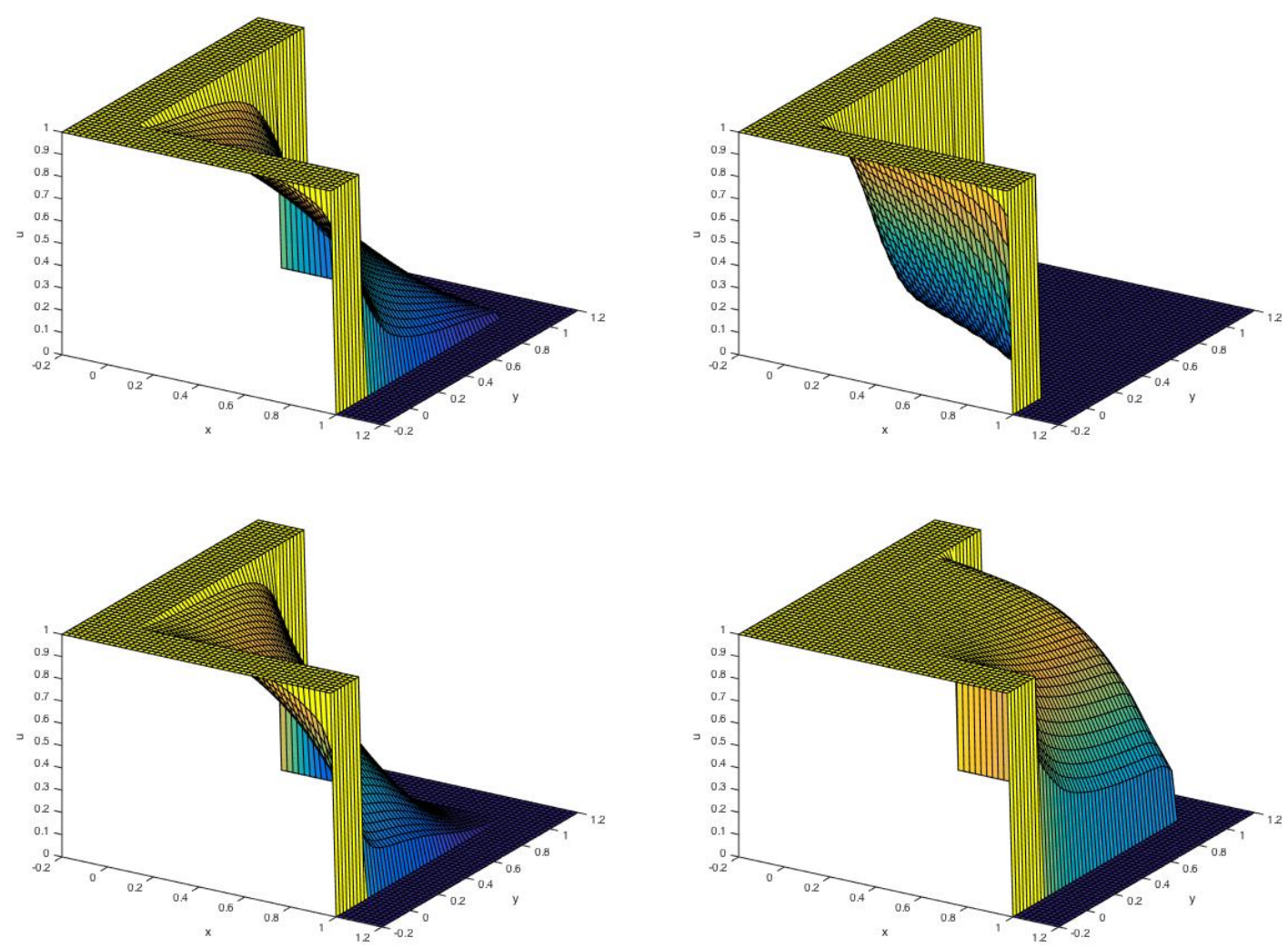

Figure 5: Approximate solutions of the strongly convection-dominated nonlocal problem $(a=$ $\left.10^{-7}\right)$ with a discontinuous velocity field in Example 3. Top-left: $\left(\theta_{1}, \theta_{2}\right)=(\pi / 4,5 \pi / 4)$; top-right: $\left(\theta_{1}, \theta_{2}\right)=(5 \pi / 4, \pi / 4)$; bottom-left: $\left(\theta_{1}, \theta_{2}\right)=(7 \pi / 4,3 \pi / 4)$; bottom-right: $\left(\theta_{1}, \theta_{2}\right)=(\pi / 4,3 \pi / 4)$.

starting from $1 / 2$ and $1 / 10$ at the coarsest grid level respectively. Tables 4.2 list numerical results on the compatibility errors measured by the $L^{\infty}$ and $L^{2}$ norms, and also convergence rates. When $a=1$, the convergence rates smoothly decrease from 2 to 1 as $\delta$ and $h$ decreases proportionally. When $a=10^{-7}$, the problem is convection-dominated and the convergence rates are basically one. These results further verify Theorem 7 , which states in (3.32) that the compatibility error for the convection term is only $O(\delta)$ when $\delta / h$ is fixed, and it dominates the convergence rate with respect to a decrease in $\delta$ and $h$ since the compatibility error for the diffusion term is $O\left(\delta^{2}\right)$.

\begin{tabular}{|c|c|c|c|c|c|c|c|c|}
\hline \multicolumn{9}{|c|}{$\boldsymbol{b}\left(x_{1}, x_{2}\right)=(\cos (\pi / 4), \sin (\pi / 4))^{T}$} \\
\hline & \multicolumn{4}{|c|}{$a=1$} & \multicolumn{4}{|c|}{$a=10^{-7}$} \\
\hline$\delta$ & $\left\|e^{h}\right\|_{\infty}$ & CR & $\left\|e^{h}\right\|_{2}$ & CR & $\left\|e^{h}\right\|_{\infty}$ & CR & $\left\|e^{h}\right\|_{2}$ & $\mathrm{CR}$ \\
\hline $1 / 2$ & $4.2744 \mathrm{e}-02$ & - & $2.1707 \mathrm{e}-02$ & - & $1.2653 \mathrm{e}-01$ & - & $5.0233 \mathrm{e}-02$ & - \\
\hline $1 / 4$ & $1.2495 \mathrm{e}-02$ & 1.77 & $6.5277 \mathrm{e}-03$ & 1.73 & $6.4556 \mathrm{e}-02$ & 0.97 & $2.4436 \mathrm{e}-02$ & 1.04 \\
\hline $1 / 8$ & $5.0913 \mathrm{e}-03$ & 1.30 & $2.5437 \mathrm{e}-03$ & 1.36 & $3.4411 \mathrm{e}-02$ & 0.91 & $1.2497 \mathrm{e}-02$ & 0.98 \\
\hline $1 / 16$ & $2.4679 \mathrm{e}-03$ & 1.05 & $1.1995 \mathrm{e}-03$ & 1.08 & $1.8384 \mathrm{e}-02$ & 0.90 & $6.3787 \mathrm{e}-03$ & 0.96 \\
\hline $1 / 32$ & $1.2905 \mathrm{e}-03$ & 0.94 & $6.6411 \mathrm{e}-04$ & 0.85 & $9.6059 \mathrm{e}-03$ & 0.94 & $3.2126 \mathrm{e}-03$ & 0.99 \\
\hline
\end{tabular}

Table 2: Numerical results on solution errors and convergence rates by the quadrature-based finite difference discretization with $\frac{\delta}{h}=5$ in Example 4 . 


\section{Conclusions}

This paper presents and analyzes a new nonlocal convection-diffusion model and its effective numerical discretization. In particular, the nonlocal convection operator is constructed through a special upwind manner. Though it may appear first that the nonlocal model involves more sophisticated definitions of nonlocal interactions, there are advantages in preserving important physical features such as the mass conservation and the maximum principle. The quadrature-based finite difference is simpler to implement than the finite element approach proposed in [43], and it also preserves the mass conservation and upwind properties at the discrete level.

It is of much practical value to develop effective mathematical models of complex transport processes for which singularity formation might be an issue of concern. By avoiding spatial differentiations explicitly, nonlocal models have distinct advantages in dealing with such singular solutions. There are still many interesting works to pursue with regard to the scheme discussed here such as effective assembly and numerical solution of the resulting algebraic systems, as well as further extensions. On the modeling side, we may consider how to effectively incorporate other boundary conditions $[8,15]$ and nonlinearity [10]. On the discretization side, our study here has focused on a particular quadrature-based finite difference scheme, It is also interesting to consider issues like effective nonlocal gradient recovery [17] and the construction of other numerical schemes, possibly of nonlinear and adaptive nature, to achieve high order accuracy while preserving the nice features of the nonlocal models.

\section{References}

[1] E. Buades, B. Coll And J. M. Morel, Image denoising methods. a new nonlocal principles, SIAM Rev., 52, pp. 113-147, 2010.

[2] F. Bobaru and M. Duangpanya, A peridynamic formulation for transient heat conduction in bodies with evolving discontinuities, J. Comput. Phys., 231, pp. 2764-2785, 2012.

[3] F. Bobaru, M. Yang, L.F. Alves, S.A. Silling, E. Askari and J. Xu, Convergence, adaptive refinement, and scaling in 1D peridynamics, Int. J. Numer. Meth. Eng., 77, pp. 852-877, 2009.

[4] J. Carrillo, R. Eftimie, and F. Hoffmann, Non-local kinetic and macroscopic models for self-organised animal aggregations, Kinetic and Related Models, 8, pp. 413-441, 2015.

[5] X. Chen and M. Gunzburger, Continuous and discontinuous finite element methods for a peridynamics model of mechanics, Comput. Methods Appl. Mech. Engrg., 200, pp. 1237-1250, 2011.

[6] O. Defterli, M. D’Elia, Q. Du, M. Gunzburger, R. Lehoucq, and M. Meerschaert, Fractional diffusion on bounded domains, Fractional Calculus and Applied Analysis, 18, pp. 342-360, 2015.

[7] M. D'Elia, Q. Du, M. Gunzburger and R. Lehoucq, Finite range jump processes and volume-constrained diffusion problems, Technical Report \#SAND 2014-2584J, Sandia National Laboratories, Albuquerque, 2014. 
[8] Q. Du, M. Gunzburger, R. Lehoucq and K. Zhou, Analysis and approximation of nonlocal diffusion problems with volume constraints, SIAM Rev., 54, pp. 667-696, 2012.

[9] Q. Du, M. Gunzburger, R. LehoucQ And K Zhou, A nonlocal vector calculus, nonlocal volume-constrained problems, and nonlocal balance laws, Math. Models Methods Appl. Sci., 23, pp. 493-540, 2013.

[10] Q. Du And Z. Huang, Numerical solution of a scalar one-dimensional monotonicitypreserving nonlocal nonlinear conservation law, J. Math. Res. Appl., 37, 1-18, 2017.

[11] Q. Du, L. Ju, L. Tian And K Zhou, A posteriori error analysis of finite element method for linear nonlocal diffusion and peridynamic models, Math. Comp., 82, pp. 1889-1922, 2013.

[12] Q. Du, Z. Huang And R. LehoucQ, Nonlocal convection-diffusion volume-constrained problems and jump processes, Dis. Cont. Dyn. Sys. B, 19, pp. 373-389, 2014.

[13] Q. Du, J. Kamm, R. Lehoucq and M. Parks, A new approach for a nonlocal nonlinear conservation law, SIAM J. Appl. Math., 72 pp. 464-487, 2012.

[14] Q. Du, L. Tian and X. Zhao, A convergent adaptive finite element algorithm for nonlocal diffusion and peridynamic models, SIAM J. Numer. Anal., 51 pp. 1211-1234, 2013.

[15] Q. Du, Y. TaO And X. Tian, Nonlocal diffusion and peridynamic models with Neumann type constraints and their numerical approximations, Appl. Math. Comp., in press, 2017.

[16] Q. Du, Y. TaO, X. Tian and J. Yang, Asymptotically compatible discretization of multidimensional nonlocal diffusion models and nonlocal Green's function, preprint, 2017.

[17] Q. Du, Y. TAO, X. TIAn And J. Yang, Robust a posteriori stress analysis for approximations of nonlocal models via nonlocal gradients, Comp. Methods Appl. Mech. Engrg., 310, pp. 605$627,2016$.

[18] Q. Du And K. Zhou, Mathematical analysis for the peridyanmic nonlocal continuum theory, ESIAM: M2AN Math. Mod. Numer. Anal., 45, pp. 217-234, 2011.

[19] V.J. ERvin and J.P. Roop, Variational solution of fractional advection dispersion equations on bounded domains in $\mathbb{R}^{d}$, Numer. Meth. PDEs, 23, pp. 256-281, 2007.

[20] G. Gilboa And S. Osher, Nonlocal linear image regularization and supervised segmentation. Multiscale Model. Simul., 6, pp. 595-630, 2007.

[21] G. Gilbon And S. Osher, Nonlocal operators with applications to image processing, Multiscale Model. Simul., 7, pp. 1005-1028, 2008.

[22] M. Gunzburger and R. B. LehoucQ, A nonlocal vector calculus with application to nonlocal boundary value problems, Multiscale Model. Simul., 8, pp. 1581-1598, 2010.

[23] W. Hu, Y. Ha And F. Bobaru, Peridynamic model for dynamic fracture in unidirectional fiber-reinforced composites, Comput. Methods Appl. Mech. Engrg., 220, pp. 247-261 2012.

[24] L. Ignat And J. Rossi, A nonlocal convection-diffusion equation, J. Funct. Anal., 251, pp. 399-437, 2007. 
[25] T. IkedA, Maximum Principle in Finite Element Methods for Convection-Diffusion Phenomena, North-Holland, Amsterdam, 1983.

[26] R. Lipton, Cohesive dynamics and brittle fracture, J. Elasticity, 124, pp. 143-191, 2016.

[27] R. Macek And S. Silling, Peridynamics via finite element analysis, Finite Elements in Analysis and Design, 43, pp. 1169-1178, 2007.

[28] M. Meerschaert, D. Benson and B. BÄumer, Multidimensional advection and fractional dispersion, Phys. Rev. E, 59, pp. 5026-5028, 1999.

[29] A. Mizukami And T.J.R. Hughes, A petrov-Galerkin finite element method for convectiondominated flows: an accurate upwind techique for satifying the maximum principle, Comput. Methods Appl. Mech. Engrg., 50, pp. 181-193, 1985.

[30] S. Oterkus, E. Madednci and A. Agwai, Peridynamic thermal diffusion, J .Comput. Phys., 265, pp. 71-96, 2014.

[31] K. Painter, J. M. Bloomfield, J. Sherratt and A. Gerisch, A nonlocal model for contact attraction and repulsion in heterogeneous cell populations, Bull. Math. Biol., 77, pp. 1132-1165, 2015.

[32] R.J. Plemmons, M-matrix characterizations. I - Nonsingular M-matrices, Linear Algebra and its Applications, 18, pp. 175-188, 1977.

[33] P. Seleson, M. Gunzburger and M. L. Parks, Interface problems in nonlocal diffusion and sharp transitions between local and nonlocal domains, Comput. Methods Appl. Mech. Engrg., 266, pp. 185-204, 2013.

[34] S. Silling, Reformulation of elasticity theory for discontinuities and long-range forces, $J$. Mech. Phys. Solids., 48, pp. 175-209, 2000.

[35] S. Silling, Linearized theory of peridynamic states, J. Elasticity, 99, pp. 85-111, 2010.

[36] S. Silling And E. Askari, A gridfree method based on the peridynamic model of solid mechanics, Comput. Struct., 83, pp. 1526-1535, 2005.

[37] S. Silling, M. Epton, O. Weckner, J. Xu and E. Askari, Peridynamic states and constitutive modeling, J. Elasticity, 88, pp. 151-184, 2007.

[38] S. Silling And R Lehoucq, Peridynamic theory of solid mechanics, Adv. Appl. Mech., 44, 73-168, 2010.

[39] S. Silling, M Zimmermann and R. Abeyaratne, Deformation of a peridynamic bar, $J$. Elasticity, 73, pp. 173-190, 2003.

[40] J. Sun, J. Li AND Q. Liu, Cauchy problem of a nonlocal $p$-Laplacian evolution equation with nonlocal convection, Nonlinear Analysis, 95, pp. 691-702, 2014.

[41] X. Tian And Q. Du, Analysis and comparison of different approximation to nonlocal diffusion and linear peridynamic equations, SIAM J. Numer. Anal., 51, pp. 3458-3482, 2013. 
[42] X. Tian And Q. Du, Asymptotically compatible schemes and applications to robust discretization of nonlocal models, SIAM J. Numer. Anal., 52, pp. 1641-1665, 2014.

[43] H. Tian, L. Ju And Q. Du, Nonlocal convection-diffusion problems and finite element approximations, Comput. Methods Appl. Mech. Engrg., 289, pp. 60-78, 2015.

[44] L. Vulkov, J. Miller And G. Shishkin, Analytical and Numerical Methods for Convectiondominated and Singularly Perturbed Problems, Nova Science Pub. Inc., 2000.

[45] H. Wang And H. Tian, A fast and faithful collocation method with efficient matrix assembly for a two-dimensional nonlocal diffusion model, Comput. Methods Appl. Mech. Engrg., 273, pp. 19-36, 2014.

[46] O. Weckner and E. Emmrich, The effect of long-range forces on the dynamics of a bar, $J$. Mech. Phys. Solids., 53, pp. 705-728, 2005.

[47] P. Wesseling, Principles of Computational Fluid Dynamics, Springer, 2000.

[48] K. Zhou And Q. Du, Mathematical and numerical analysis of linear peridynamic models with nonlocal boundary condition, SIAM J. Numer. Anal., 48, pp. 1759-1780, 2010. 